\title{
Influence of Interfacial Interactions on Deformation Mechanism and Interface Viscosity in $\alpha$-Chitin-Calcite Interfaces
}

\author{
Tao $\mathrm{Qu}^{1}$, Devendra Verma ${ }^{1}$, Milad Alucozai ${ }^{1}$, and Vikas Tomar ${ }^{1 *}$ \\ ${ }^{1}$ School of Aeronautics and Astronautics, Purdue University-IN, 47907, USA \\ *Corresponding Author, Email: tomar@purdue.edu, Tel: 765-494-3423
}

\begin{abstract}
The interfaces between organic and inorganic phases in natural materials have a significant effect on their mechanical properties. This work presents a quantification of the interface stress as a function of interface chemical changes (water, organic molecules) in chitin-calcite (CHI-CAL) interfaces using classical non-equilibrium molecular dynamics (NEMD) simulations and steered molecular dynamics (SMD) simulations. NEMD is used to investigate interface stress as a function of applied strain based on the virial stress formulation. SMD is used to understand interface separation mechanism and to calculate interfacial shear stress based on a viscoplastic interfacial sliding model. Analyses indicate that interfacial shear stress combined with shear viscosity can result in variations to the mechanical properties of the examined interfacial material systems. It is further verified with Kelvin-Voigt and Maxwell viscoelastic analytical models representing viscous interfaces and outer matrix. Further analyses show that overall mechanical deformation depends on maximization of interface shear strength in such materials. This work establishes lower and upper bounds of interface strength in the interfaces examined.
\end{abstract}

Keywords: Steered Molecular Dynamics, Chitin, Calcite, Interfacial Stress, Shear Viscosity, Interface Viscosity 


\section{\$1 Introduction}

Interfaces significantly dominate mechanical properties of biomaterials [1]. Biomaterials such as marine crustacean exoskeleton, bone, and nacre are composed of a hierarchy of interfaces with an organic phase (e.g. chitin (CHI) or tropocollagen (TC)) and a mineral phase (e.g. calcite (CAL) or hydroxyapatite (HAP)) arranged hierarchically at the structural length scale of $n m$.

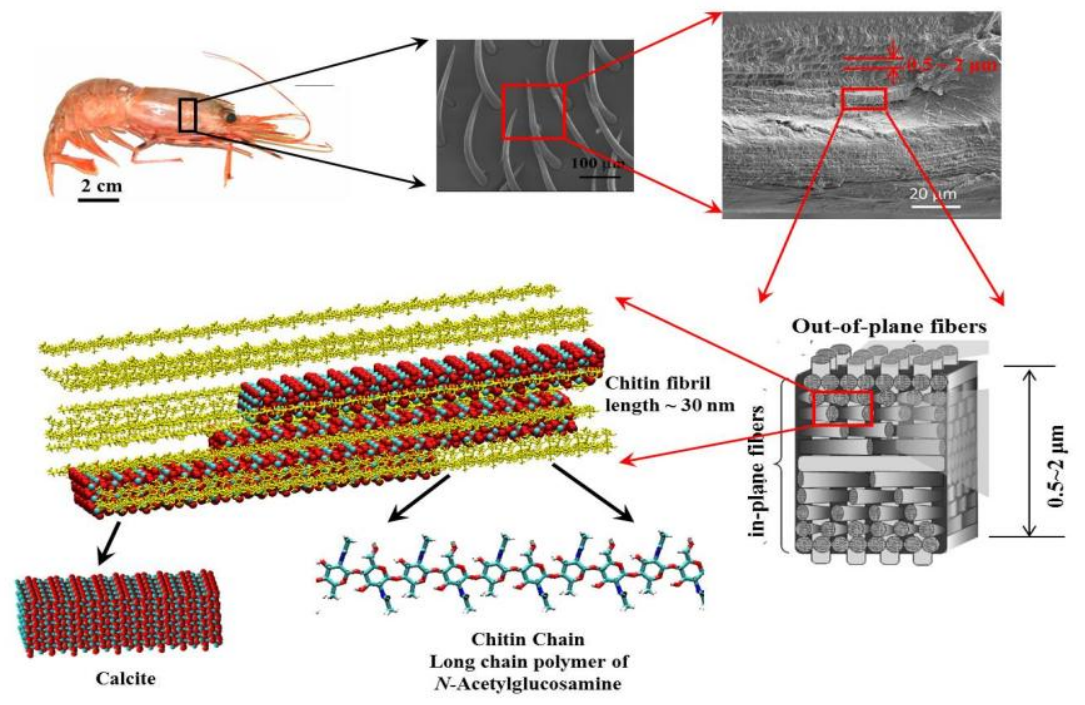

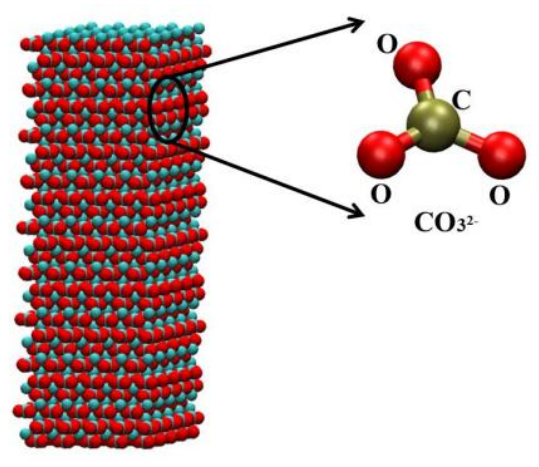

(b)

(a)

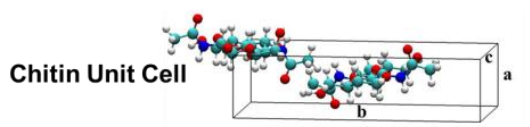

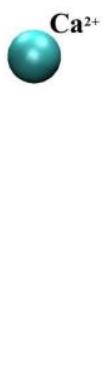

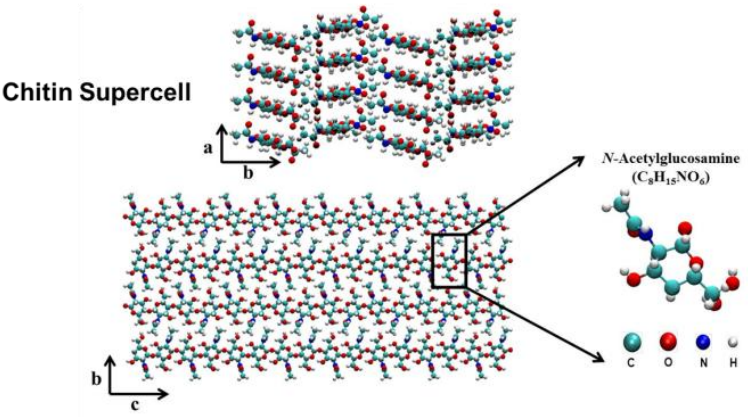

(c)

Figure 1. Schematic of the hierarchical structure of a marine crustacean exoskeleton. 
Structural studies of such biological materials have shown that at mesoscale $(\sim 100 \mathrm{~nm}$ to few $\mu \mathrm{m})$, the mineral crystals are preferentially aligned along the length of organic phase polypeptide molecules in a hierarchical (e.g. staggered or Bouligand pattern) arrangement as shown in Fig. 1. Studies also show that such staggered hierarchical structure leads to a unique combination of toughness and strength properties. Interfaces play a significant role in the stress transfer resulting in improved stiffness and strength of such material systems. However, how exactly the change in interfacial chemical configuration leads to change in interface mechanical properties need further investigation. Current study is focusing on the calcite-chitin interactions, which widely exist in exoskeleton materials [2] but have not been studied in detail before. With this view the present work focuses on quantifying interface stress as a function of interface chemical changes (water, organic molecules) in selected chitin-calcite (CHI-CAL) interfaces using classical nonequilibrium molecular dynamics (NEMD) simulations and steered molecular dynamics (SMD) simulations. One important step in understanding the relationship between interfaces and material properties is the knowledge of the fundamental mechanisms guiding stress transfer across the organic-inorganic interfaces. Therefore, this work characterizes the interaction between organic-inorganic interfaces as a function of mechanical straining. The present work is based on the use of NEMD and SMD simulations and therefore does not consider the effect of factors such as quasi-static loading conditions, structural gradients, presence of other organics and hydration, longer length-scales, etc that cannot be addressed using molecular simulations due to fundamental limitations of such. However, the present work presents mechanistic insights behind interface behavior that are not accessible using experiments. The presented results should be considered in light of such interactions. 
Experimental techniques such as electron microscopy, X-ray diffraction, indentation, and tensile (or compressive) testing have previously been performed to characterize the structural and mechanical properties (including hardness, modulus, elastic-plastic deformation etc.) of chitin based materials such as cuticles, exoskeletons from prawn, crab and lobsters (Guille et al. [3, 4], Roer and Dillaman [5], Raabe et al. [6-8], Al-Sawalmih et al. [2], Joffe et al. [9], Vincent and Wegst [10], Sachs et al. [11, 12], Fabritius et al. [13]). Previous analytical work includes studies based on the tension shear chain model developed by Gao and coworkers [14, 15]. Atomistic simulations have been widely used to investigate the mechanical behavior of biomaterials, especially bone, with the goal of understanding the role of organic phases (i.e. TC in bone) and inorganic phases (i.e. HAP in bone) [16-19]. However, atomistic studies on chitin based materials have been limited. Jin et al. [20] studied mechanical properties of the chitin-protein interface by performing equilibrium molecular dynamics (EMD) simulations. Nikolov et al. [21, 22] reported the stiffness tensor of single crystalline chitin by performing ab-initio simulations. They predicted effective properties of chitin-protein fibers with a mean-field homogenization method. Molecular mechanics-based studies on the interfacial interactions in a multiphase composite system have also been performed using SMD to study the interfaces in collagen based materials by peeling off collagen filaments from the HAP substrates [23-25].

Despite such significant advances mentioned above, molecular-simulation-based studies aimed at explicitly calculating interface stress in biological and polymeric systems have been limited. Lindal and Edholm [26] introduced a computational method to decompose the net surface tension in polymeric membrane systems simulated using NEMD. A viscoplastic model has also been used by Frankland and Harik [27] to characterize the sliding process between carbon nanotubes and epoxy based polymer matrix. Buehler [28] studied the homogeneous shear 
and slip pulses process between collagen fibrils using MD simulations based on a onedimensional model of fracture. Dubey and Tomar [29] performed 3-D ab initio MD (AIMD) simulations to understand atomic interactions in TC-HAP interfaces under tensile loading. However, AIMD simulations to date have limited length scales. The framework developed in this work to understand interface deformation mechanics using SMD is based on a combination of frameworks reported by Katti and coworkers [23-25], Lindal and Edholm [26], and Frankland and Harik [27]. Similar molecular mechanics framework has been incorporated with the continuum micromechanics to top-down access to interface viscosity of bone material systems in the earlier studies [30-33]. NEMD simulations to evaluate interface strength are based on the pressure profile calculation method, [26]. The shear interactions between the inorganic (CAL) and organic $(\mathrm{CHI})$ phases are characterized using SMD simulations performed to initiate the interfacial sliding process. A viscoplastic model is then used to calculate the shear yield stress and shear viscosity of the interfacial material systems.

\section{\$2 Method and Framework}

All NEMD and SMD supercells analyzed in the present work had organic fibers (CHI molecules) aligned parallel to the surface of inorganic crystals (CAL molecules).

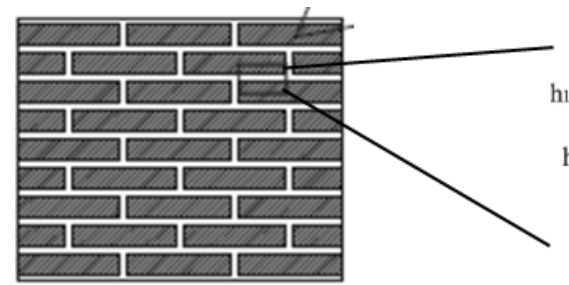

Mineralized fiber

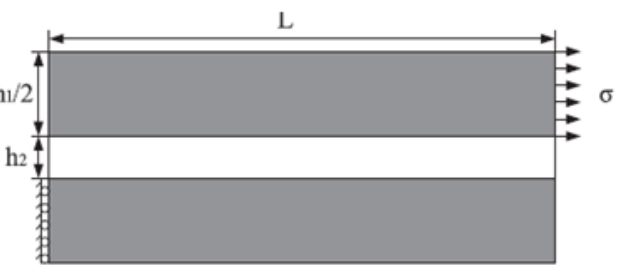

Unit cell

Figure 2. Schematic of 2D Biocomposite and 2D Simplified Unit Cell 
The $c$-axis of the trigonal CAL crystals is along the $z$-axis in the system. According to available previous studies [34-36], this configuration corresponds to the simplified unit cell structure shown in Fig. 2. 3-D NEMD and SMD simulations were performed on the interfaces modeled by embedding the organic molecular systems (i.e. CHI) between the inorganic molecular systems (i.e. CAL). Water (WT) molecules were added to the interface region to analyze the effect of hydration on interface stress. Interfaces of chitin based materials analyzed in the present work are shown in Figs. 3(a) to (d) (denoted as CAL-CAL, CAL-WT-CAL, CALCHI-CAL, and CAL-CHI-WT-CAL supercells, respectively). The interface separation in Figs. 3(c) and 3(d) was chosen in order to have one layer of $\mathrm{CHI}$ molecules in the interface region. The effect of changes in thickness of interfaces on interface mechanics will be the focus of a future investigation. Load was applied in the $x$-axis direction for CAL-CAL and CAL-WT-CAL supercells and in $x$ - and $y$-axis directions for CAL-CHI-CAL and CAL-CHI-WT-CAL supercells, indicated in Fig. 3(e). Simulations with the loading direction in z-axis were also performed. However, the current paper is focusing on shear interactions and interfacial sliding process (shown in Fig. 2) and the results under the z-axis loading condition will be the focus of a future investigation. A full model of the CHI-based biomaterials should include CHI chains, binding proteins, small amounts of amorphous calcium phosphate (ACP), amorphous calcium carbonate (ACC), as well as crystalline calcite $[2,20]$. Due to the complexity of performing atomistic analysis of supercells, attention is limited to analyzing the behavior of the two main components, $\mathrm{CHI}$ fibers and crystalline CAL. CHI is a long chain polymer of $\beta-1,4-N$-acetyl-Dglucosamine (GlcNAc) and is the second most abundant biological material on the earth after cellulose. Chitin exists in three different polymorphic forms that differ in the arrangement of chitin molecular chains in the crystal cell [37]. 


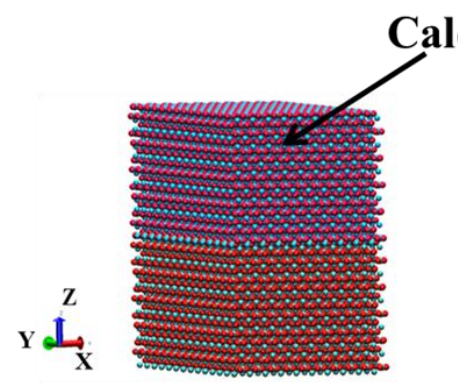

(a)

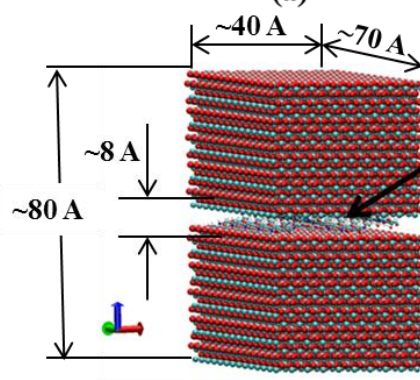

(c)

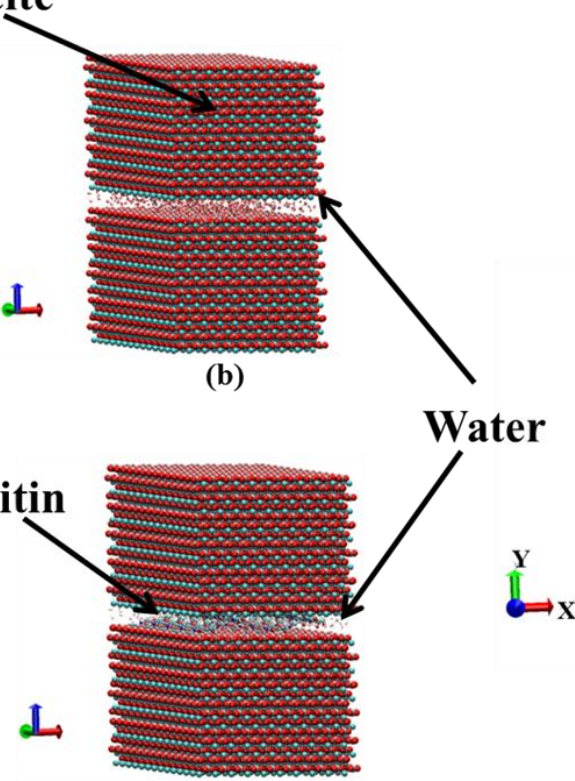

(d)
Loading condition X

\&

Loading condition Y

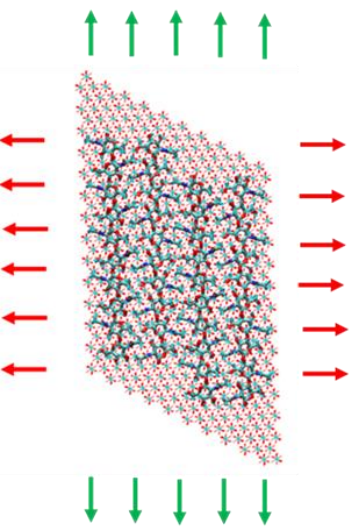

(e)

Figure 3. A schematic showing of the chitin based material system being analyzed (a) CALCAL (b) CAL-WT-CAL (c) CAL-CHI-CAL (d) CAL-CHI-WT-CAL (e) schematic to show the loading direction

The chains are arranged in antiparallel fashion in the case of $\alpha-\mathrm{CHI}$ while chains are parallel in $\beta$-CHI, and $\gamma-\mathrm{CHI}$ is a mixture of $\alpha$ - and $\beta$-CHI. $\alpha-\mathrm{CHI}$ was chosen for this study since it is mainly found in marine crustacean exoskeleton and is more stable and more abundant than the other two [8, 38]. It was determined that the crystalline $\alpha-\mathrm{CHI}$ (shown in Fig. 1(c)), $\left(\mathrm{C}_{8} \mathrm{H}_{13} \mathrm{O}_{5} \mathrm{~N}\right)_{\mathrm{n}}$, is orthorhombic lattice structure with $\mathrm{P} 2{ }_{1} 2_{1} 2_{1}$ symmetry [39]. The present model was built based on the recently-reported crystallographic constants [40, 41], $a=4.74 \AA$, $b=18.86$ $\AA, c=10.32 \AA$ and $\alpha=\beta=\gamma=90^{\circ}$. The calcite supercell (shown in Fig. 1-(b)) was generated using fractional coordinate and unit cell parameter information given in Reference [42], which is a trigonal crystal system with space group R3c, $a=b=4.989 \AA, c=17.062 \AA, \alpha=\beta=90^{\circ}$ and $\gamma=120^{\circ}$. The calcite supercell consisted of 420 unit cells with 25 atoms per unit cell and one CHI molecular chain consists of 270 atoms. 
The NAMD package, [43], was used to perform both NEMD and SMD simulations. Water molecules in the interface region were added using 'SOLVATE' module in the visual molecular dynamics (VMD) software, [44]. For chitin based materials, a combination of CHARMM36 carbohydrate force field, [45], and CHARMM36 force field for small molecule drug design, [46], was used to model the atomistic interactions. Geometric mean was used for van der Waals parameters in order to define atomistic interactions at the CHI-CAL interfaces. The CHARMM36 carbohydrate force field and topology parameters for chitin molecules, CHARMM36 small molecule drug design force filed and topology parameters for calcite molecules were directly obtained from the CHARMM website, $[45,46]$. In all simulations, the particle mesh Ewald method was used to calculate electrostatic interactions with a cut off of 12 $\AA$. All simulations were performed using the NAMD package as it works better with large biomolecules and scales well to a very large processor counts. Material systems were equilibrated using two different ensembles: (1) isothermal-isobaric (NPT) ensemble for equilibration at $1 \mathrm{~atm}$ and at $300 \mathrm{~K}$ till the volume of the supercell being equilibrated was stabilized and there were minimal pressure fluctuations; and (2) canonical (NVT) ensemble for equilibration at $300 \mathrm{~K}$ until the fluctuations in the temperature subsided. Simulations were performed with periodic boundary conditions (PBC) imposed on the supercells shown in Figs. 3.

At this point it is important to remember that interfaces considered in this work are idealized in order to systematically delineate the influence of various structural factors pointed above.

\section{\$2.1 Pressure Profile Calculations during NEMD Simulations}

Stress-strain curve was generated based on the well-known virial stress formulation. There are two loading directions (Fig. 4-(a)): direction along the molecule length ( $x$-axis) and direction transverse to molecule length ( $y$-axis). The equilibrated supercells were stretched with a strain 
increment of $\varepsilon=2 \%$ in the designated direction until $20 \%$ tensile strain level was reached. The supercell was compressed by $v \varepsilon \%$ in the transverse directions to account for Poisson's ratio (v) effect. Initially, a fixed value of $v(=0.3)$ was used based on average of the reported literature values for $\mathrm{CHI}$ based materials [21, 22]). Afterwards, the supercell was equilibrated and actual Poisson's ratio for the supercell automatically arose out of transverse atomic displacements during supercell equilibration. The supercell being analyzed was then equilibrated using the same sequence of NPT-NVT equilibration as that at $0 \%$ strain. The 'pressure Profile' option was turned on and 'pressure Profile Slabs' was chosen as 11 for chitin based material systems, Fig. 4(b). NAMD supports the calculation of the pressure profiles as a function of the $z$-coordinate in the system based on the algorithm developed by E. Lindahl and O. Edholm [26]. The simulation system was divided into slabs and three diagonal components of the pressure tensor in each slab were given in output. The virial stress tensor of each slab and the overall system at the end of equilibration was recorded as the stress tensor corresponding to each strain level until 20\% strain was achieved. The effective elastic moduli were obtained by linear fitting of the stress-strain curve for each material system. These procedures made it possible to estimate how the measured stress of the loaded material system was distributed inside its interfacial regions and the stress between the interfaces was obtained according to the behavior of corresponding slab of the simulation system, Fig. 4-(b). 


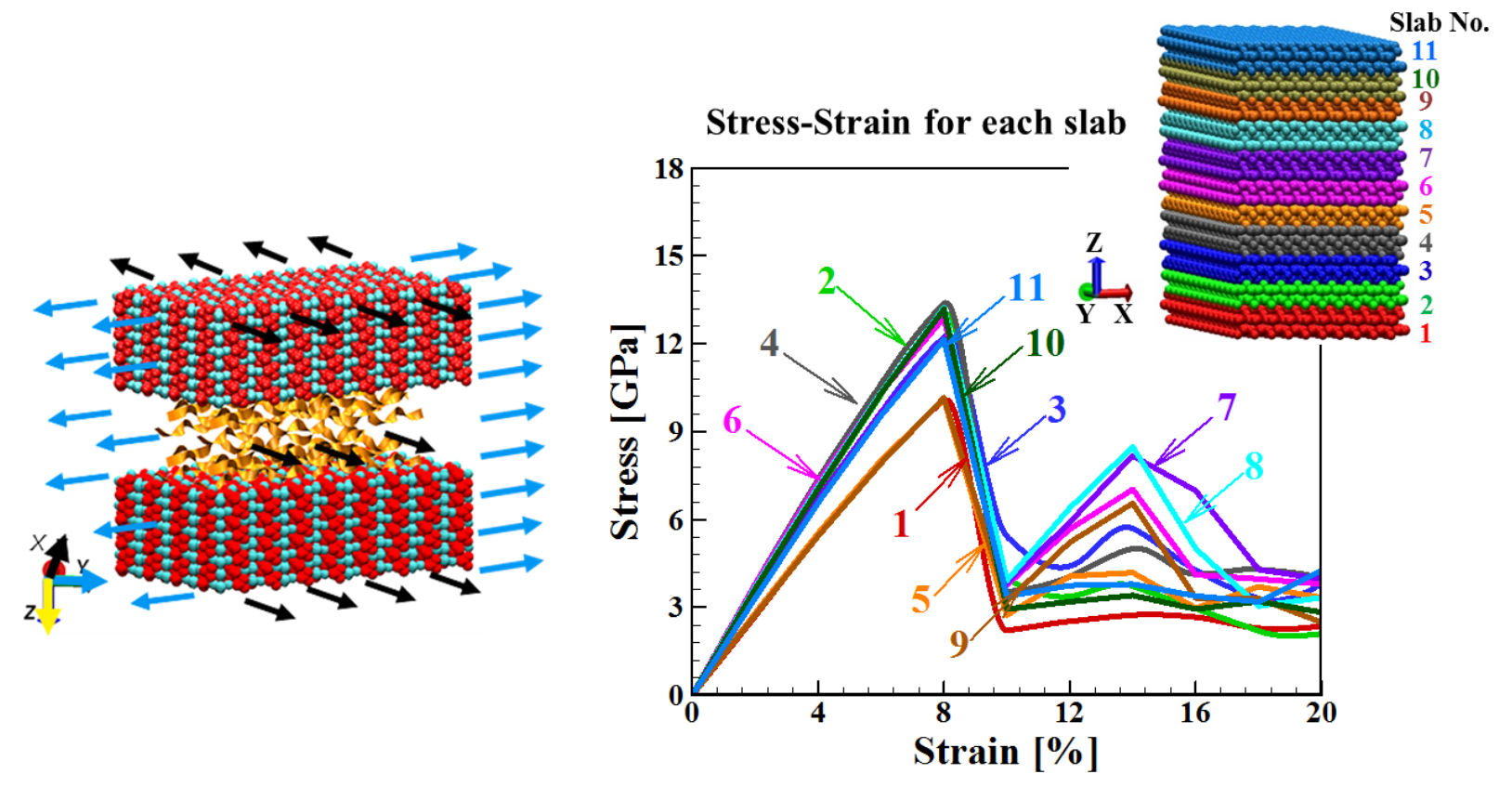

Figure 4 (a) A schematic showing of loading condition of the interfacial material system, (b) stress-strain curve for each slab of the system from the bottom to the top layer.

\section{\$2.2 SMD Simulation Setup}

SMD simulations in the constant speed mode, [43], (Fig. 5-(a)) were used to pull out the upper inorganic crystals (CAL) from the substrate inorganic crystals (CAL) to replicate the interfacial sliding process. There are two loading directions (Fig. 5-(a)): direction along the molecule length ( $x$-axis) and direction transverse to molecule length (y-axis). SMD force was applied to the center of mass of upper inorganic crystals in a chosen direction. The organic molecules $(\mathrm{CHI})$ and water molecules in the interface region were not constrainted. The substrate inorganic crystals were fixed on the bottom. Lagevine dynamics was used for temperature control. PBC was applied in the $x-y$ plane, Fig. 3, [47]. 

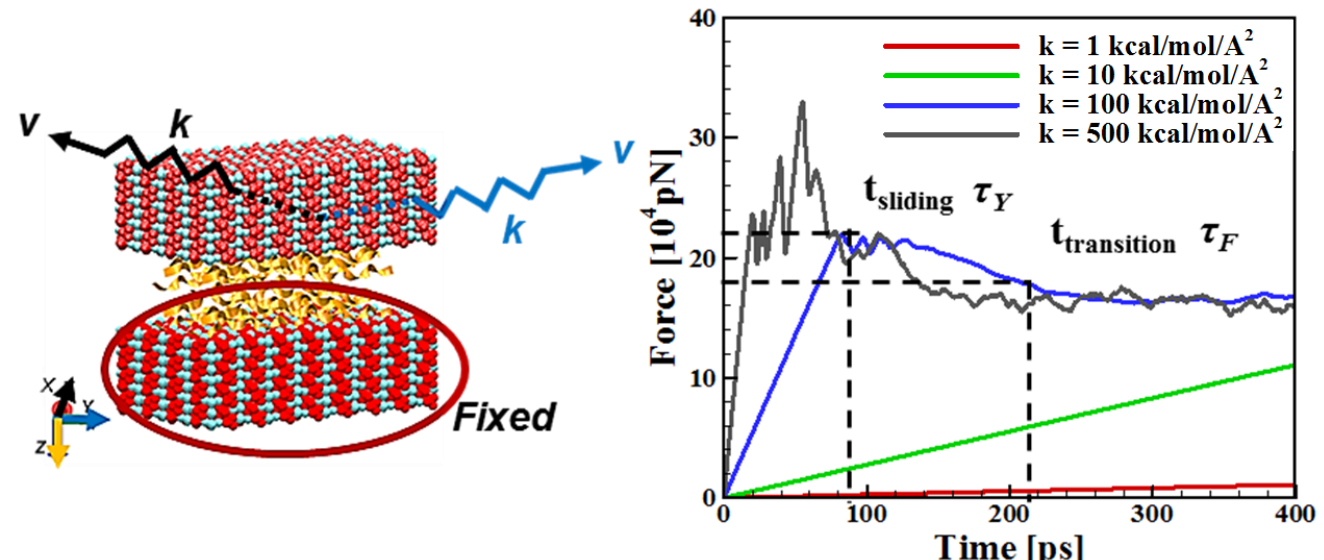

(a)

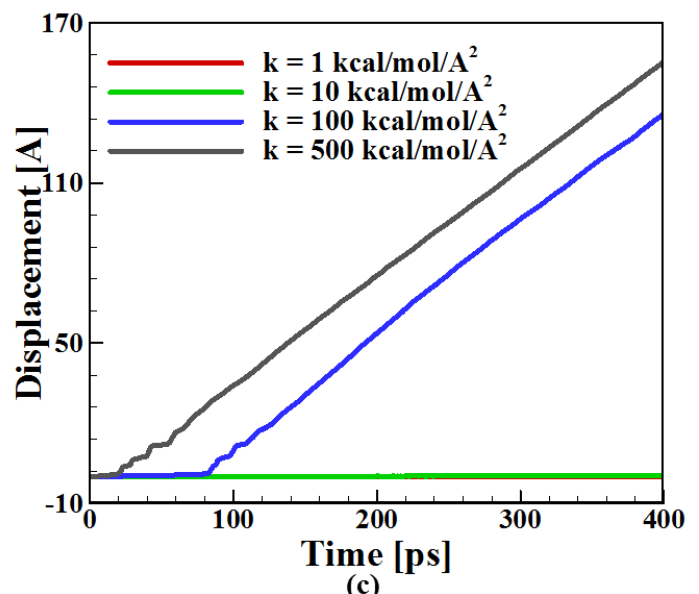

(b)

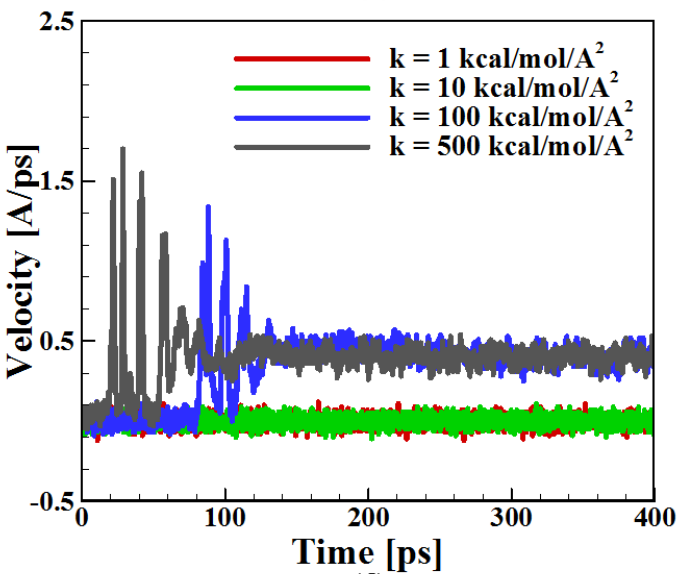

(d)

Figure 5. (a) Schematic showing of loading condition of the SMD in constant speed pulling mode, (b) SMD force as a function of time, (c) displacement of the mass center of the SMD structure as a function of time, (d) velocity of the mass center of the SMD structure as a function of time.

Four simulations were performed with varying spring constants $k(=1,10,100,500$ $\mathrm{kcal} / \mathrm{mol} / \mathrm{A}^{2}$ ) to determine the appropriate spring constant for each system. The spring constant must be high enough to enable local unbinding potential but should not be so high as to control the background noise within the specified limits, [48]. The suitable pulling velocity $\mathrm{v}$ should be as small as possible according to an earlier study, [48]. Velocity v=0.4 $\AA$ /ps was chosen for this study. The force and displacement of the SMD structures (upper inorganic crystals) from the output were plotted as a function of time (Figs. 5 (b) and (c)). Structural velocity as a function of 
time was plotted by calculating the numerical derivative of the displacement profile (Fig. 5-(d)). The plots suggest that smaller spring constants produced a delay in the peak force value and spring constants of 1 and $10 \mathrm{kcal} / \mathrm{mol} / \AA^{2}$ were not enough to initiate the slippage between the SMD structures and the fixed structures. Based on the plots, the entire interfacial sliding process, for $k=100 \mathrm{kcal} / \mathrm{mol} / \AA^{2}$ in Fig. 5, can be described in the following four stages:

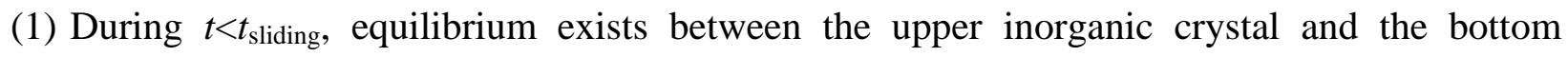
inorganic crystal.

(2) At $t=t_{\text {sliding, }}$, the applied force reaches the critical force, $F_{0}$ and the yield stress, $\tau_{\mathrm{Y}}$ that initiates the slippage between the SMD and fixed structures. This onset of slippage is marked by a noticeable increase in the displacement and the velocity of the SMD structures.

(3) During $t_{\text {sliding }}<t<t_{\text {transition, }}$, the sliding fully develops into a constant velocity stage. In this stage, the velocity of the SMD structure fluctuates and the force slowly reduces to a constant value.

(4) During $t>t_{\text {transition, during the steady state sliding process the shear force generated between }}$ the interfaces equals the applied SMD force. The interfacial shear stress denoted as the failure stress, $\tau_{\mathrm{F}}$, can be calculated with the applied force and the interfacial area.

\section{\$2.3 Viscoplastic Interfacial Sliding Model}

A viscoplastic model, [27], for interfacial sliding was introduced to quantify the interface sliding process and failure in interface region. After the yield stress (or critical force), $\tau_{\mathrm{Y}}\left(\right.$ or $\mathrm{F}_{0}$ ), is reached, the viscoplastic failure of the interfaces is related to applied shear stress, $\tau$, and shear velocity gradient (rate of shear deformation), $\frac{\partial V}{\partial d}$, as

$$
\tau=\tau_{0}+\mu \frac{\partial V}{\partial d}
$$

Here, $\mu$ is the shear viscosity of interfacial sliding and $d$ is defined in Fig. 6 (c). 


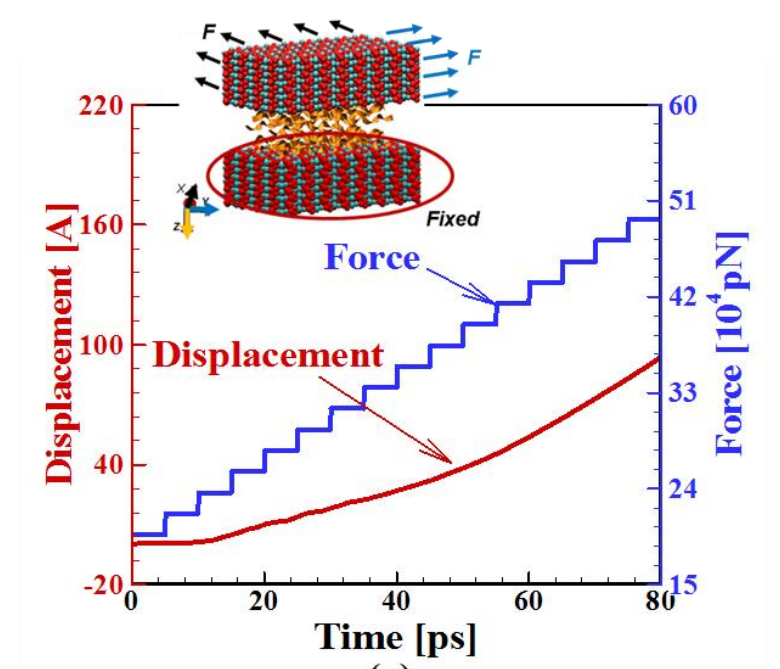

(a)

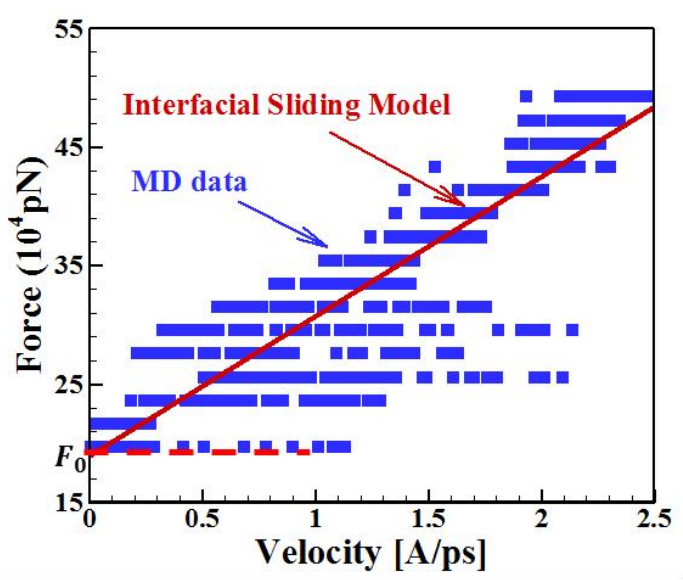

(b)

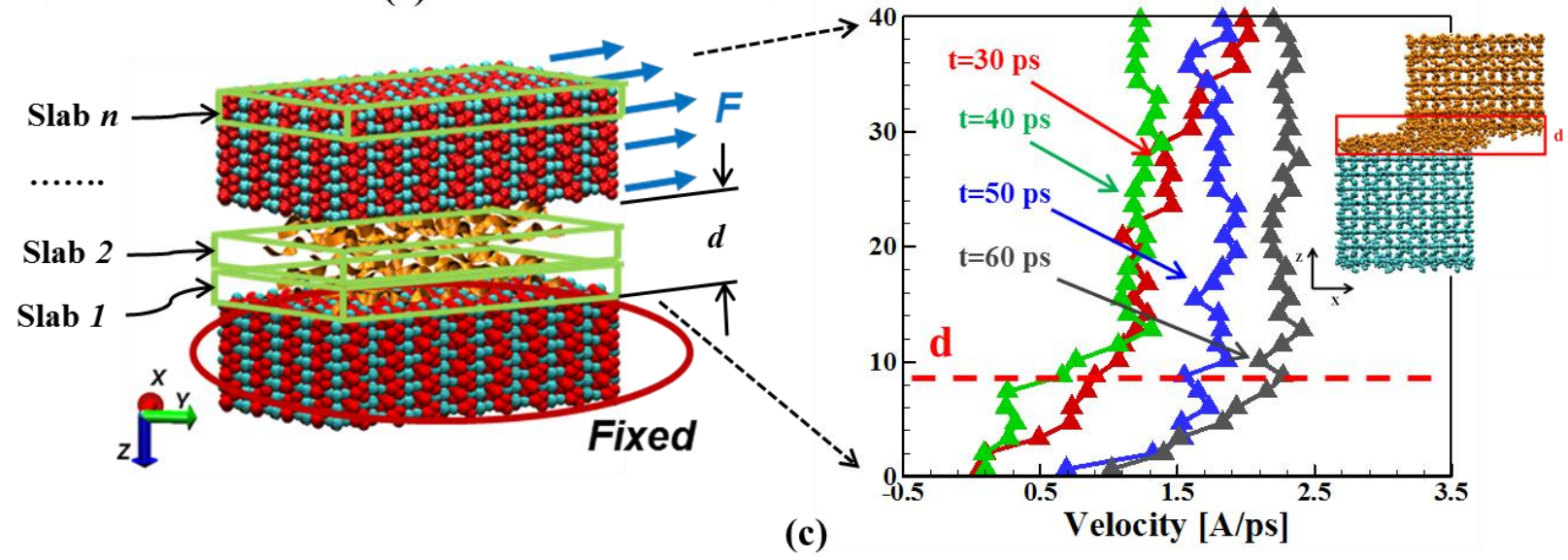

Figure 6 (a) SMD force and displacement of the SMD structure as a function of time with a schematic showing of loading condition of the SMD in constant force pulling mode, (b) curve fitting of the MD data with the visco-plastic model, (c) velocity profile at different time step.

Differing from the SMD simulation in the constant speed mode, force was applied to each atom of the upper inorganic crystal (CAL) in the designated direction. The applied force was increased incrementally over time. The starting force was smaller than the critical force determined from the simulations based on framework discussed in the earlier section. The organic molecules $(\mathrm{CHI})$ and water molecules in the interface region were free to move. The substrate inorganic crystals (HAP or CAL) were fixed on the bottom. Lagevine dynamics was used for temperature control, with PBCs applied in the $x-y$ plane, [47]. The simulation was run 
for $\sim 5000$ steps at each force level. The force and displacement as a function of time are plotted in Fig. 6(a). As an example, the shear viscosity of the CAL-CAL interface (Fig. 6) is calculated as $0.037 \mathrm{~Pa}$ s. The viscosity of the slurries of HAP was reported as $\sim 0.01-1.6 \mathrm{~Pa} s$ earlier by experiments performed by [49].

Eq (1) of the interfacial sliding model is multiplied by the interfacial area to relate shear viscosity $\mu$ to the applied force $F$ and the critical force $F_{0}$ as

$$
F=F_{0}+\mu A \frac{\partial V}{\partial d}
$$

The shear velocity, $\frac{\partial V}{\partial d}$, is the velocity gradient derived from the SMD simulation. Eq (2) can be simplified as

$$
F=F_{0}+\mu A \frac{V}{d}=F_{0}+\frac{\mu A}{d} V=F_{0}+k V .
$$

The velocity of the system, $V$, is linearly related to the applied force, $F$. Eq (3) is the interfacial sliding model used in our study. Parameter $d$ is the thickness of the interface region. The critical force $F_{0}$ and the slope $k$ can be obtained by fitting the MD data using the model given in Eq (3) (Fig. 6-(b)). The shear viscosity, $\mu$, can be described as

$$
\mu=\frac{k d}{A} .
$$

The crystal system was subdivided into slabs along the $z$-coordinate wherever force was applied to determine the velocity gradients. The displacement of each slab at every time step, $x(t)$, was measured as the position of the mass center of corresponding slab in the direction of applied force as

$$
x(t)=\frac{\sum_{i=1}^{n} m_{i} x_{i}(t)}{\sum_{i=1}^{n} m_{i}} .
$$


The velocity profile of each slab was obtained by calculating the numerical derivative of the displacement for the corresponding slab. The velocity as a function of z-coordinate at different time steps is plotted as shown in Fig. 6-(c). The thickness of the interface region, $d$, can be determined from the plot. The yield stress, $\tau_{Y}$, is calculated from the critical force, $F_{0}$, obtained by the curve fitting, and the interfacial area, $A$. The shear viscosity $\mu$, is calculated using Eq (4) with the curve fitting parameter, $k$, and the thickness of the interface region, $d$.

\section{§3 Results, Analyses, and Discussion}

\section{§3.1 Interface Effect on the Overall Mechanical Deformation Behavior of the Analyzed CHI-CAL System}

It has been shown that the failure of organic-inorganic bio-interfaces is a result of combined tensile and shear loading [17]. Visual deformation analyses have revealed that calcite and chitin portions individually develop different levels of individual strains corresponding to the uniformly imposed external strain. These factors together with the observation of ductile failure combined with visual deformation analyses, led us to believe that shear stress plays an important role in the failure of CHI-CAL composite supercells. Accordingly, the effective strength and stiffness of the supercells under study needs to be characterized using a measure based on principal stresses that can effectively describe ductile material failure with an account of shear stress dominance. We chose the widely accepted von Mises stress as such measure. The von Mises stress values were calculated based on the stress tensor components obtained from the simulations. These values were compared with the virial stress values in the loading direction and the maximum shear stress for each analyzed interfacial material system, [29]. The von Mises stress, virial stress in the loading direction, the maximum principal shear stress inside the interface region of each system and the von Mises stress distribution along z-coordinate of each 
system can be obtained based on the pressure profile calculation method outlined earlier in section 2.1 .

The elastic constant of the calcite crystal has been reported in the range of $130-150 \mathrm{GPa}$ [50-52]. This value is reduced as the organic interface (i.e. CHI) is employed to the analyzed material systems in present study since $\mathrm{CHI}$ is less stiffer than calcite, i.e. the modulus of chitin nanofibril is reported as $\sim 119 \mathrm{GPa}$ along the axial direction and the modulus in its transverse direction is only $28 \mathrm{GPa}$ [22]. The simulation systems in present work are built by attaching two CAL crystals together (or embedding the organic phases inside) which introduces the interface. Shear interaction between the interfaces is involved in deformation of the material, which leads to the non-uniform stress distribution along the z-coordinate of the material system and reduces the mechanical strength.

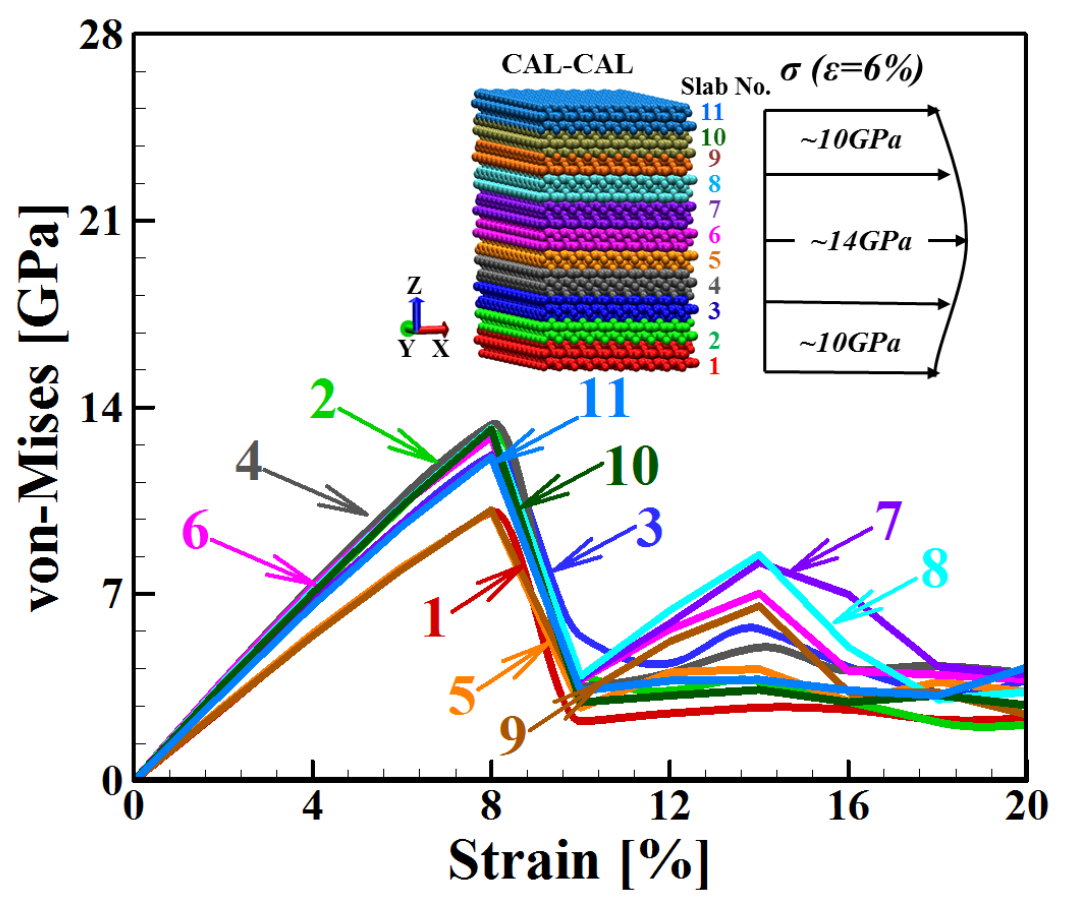

Figure 7. Plot of the von-Mises stress-strain curve for each slab of the CAL-CAL system. Image on the upper left corner shows the CAL-CAL system with the 11 slabs along z-axis from bottom to top and the stress distribution along the $\mathrm{z}$-coordinate. 
Stress distribution (at $\varepsilon=6 \%$ ) along the z-coordinate of the CAL-CAL system shown in Fig. 7 indicates that higher load is shared by the interface region compared to the top and bottom regions. A strain value of $6 \%$ was chosen as the value corresponding to elastic deformation. The stiffness of the pure inorganic system (CAL-CAL) was found to be higher than that of the interfacial systems with organic phases (or water). Besides change in the atomistic interaction magnitude, the systems with organic phases (or water) also leads to reduced interfacial contact area. It has been shown that reduced interfacial contact area leads to lower shear resistance contribution to the interfacial strength, [29]. Furthermore, the non-bonded energy between the inorganic systems is much higher than that between the organic and inorganic systems. This is verified using SMD simulations in section 3.2. This effect of hydration environment on the mechanical behavior of biomaterial systems, such as bone has been investigated in earlier studies, [29]. The focus of the present study is on understanding how water molecules affect interface strength. In contrast to previous studies, the present work only focuses on the influence of adding water in interfaces. At this point it is important to remember that interfaces considered in this work are idealized in order to systematically delineate the influence of various structural factors pointed above. Presence of other organics, longer length scales, and quasi-static loading conditions are not considered.

In the material systems where the brittle inorganic phase (CAL) occupies much larger volume fraction, normal stress (virial stress) dominates the deformation mechanism. However, shear has a significant effect on the strength of the organic phase as well as the overall interfacial mechanical behavior. Such shear effect becomes more significant as the interfacial constituent or the hydration condition varies [28, 53, 54]. Fig. 8 displays the interface level von Mises stress, virial stress in the loading direction and the maximum principal interface shear stress combined 
with the stress distribution plot as a function of length along z-axis (at $\varepsilon=6 \%$ for un-hydrated systems and at $\varepsilon=20 \%$ for hydrated systems) for the CHI-CAL interfaces analyzed in the present. The strain values were arbitrarily chosen as the value corresponding to elastic deformation. System level principal maximum shear stress (grey curve) is also plotted for comparison. Interface maximum principle shear stress (blue curve) in the hydrated systems (Fig. 8-(c) and (d)) is higher than that in the un-hydrated systems (Fig. 8-(a) and (b)). Moreover, the interface principal shear stress inside the interface region (blue curve) is closer to the overall system shear stress (grey curve) in hydrated systems (Fig. 8-(c) and (d) compared to un-hydrated systems Fig. 8-(a) and (b)). It can be concluded that the shear deformation of the material system comes primarily from the shear interaction inside the interface region. The presence of water strengthens the effect of the interfacial shear in determining the mechanical behavior of the material. The plasticizing effect of water on collagen based materials has also been observed and is predicted to be the primary contributor to this phenomenon, [16, 18, 29, 53, 55], however, the toughening mechanisms on collagen and chitin are different. In hydrated collagen, water intercalates into the structure of the fiber which can reduce the Young's modulus and enhance the shear interactions of the phases [56]. The $\alpha$-chitin structure exhibits 3D hydrogen bonding pattern and the hydrophobic faces of the carbohydrate rings stack on top of one another [37], and the hydration on chitin causes it to swell like a gel, observed in experimental studies [57]. Because the structure of $\alpha$-chitin exhibits both significant hydrogen bonding and hydrophobic interactions, the addition of the water molecules will have the stiffening effect on the chitin fibers [37, 57]. This stiffening effect is verified by the stress-strain curve shown in Fig. 8-(c) and (d), where both of the stiffness and strength measured by the interface level von-Mises stress are 
higher than ones shown in Fig. 8-(a) and (b). Next, the stiffening effect on the overall mechanical behavior of the interfacial structures will be explained further.
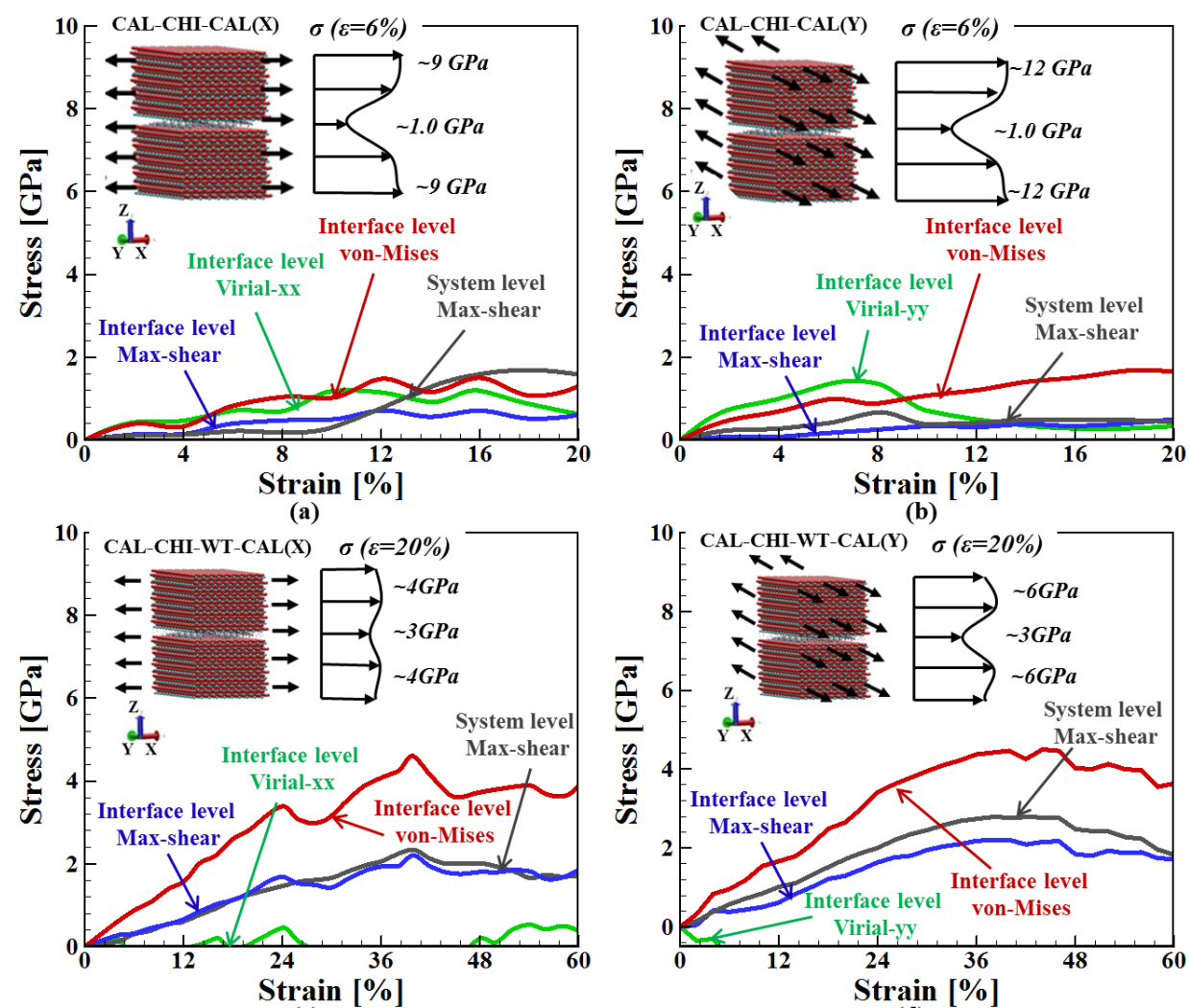

(c)

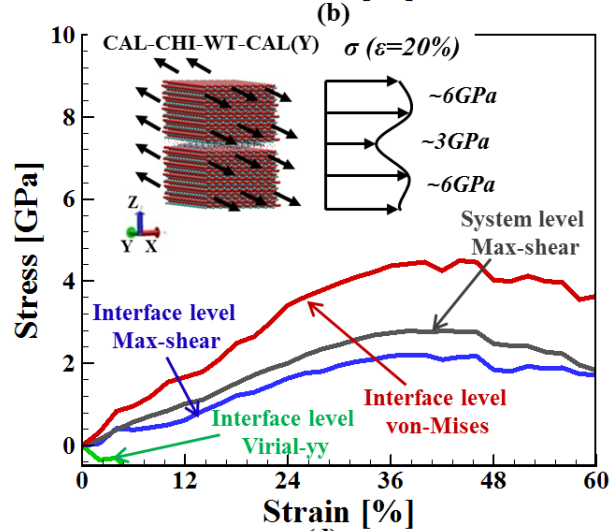

(d)

Figure 8. Plot of the von-Mises (red), virial (green), and maximum shear stress (blue) inside the interface region (interface level) as well as the overall (system level) maximum shear stress (grey) as a function of strain for the system combined with the stress distribution based on the pressure profile calculation along the $z$-axis for each system (a) CAL-CHI-CAL loaded in $x$-axis, i.e. fibril direction, (b) CAL-CHI-CAL loaded in $y$-direction, i.e. transverse fibril direction, (c) CAL-CHI-WT-CAL loaded in $x$-axis, i.e. fibril direction, (d) CAL-CHI-WT-CAL loaded in $y$ direction, i.e. transverse fibril direction.

Fig. 9 displays (system level) von Mises stress, virial stress in the loading direction, and the overall maximum principal shear stress combined with the stress distribution plot as a function of length along z-axis of the same systems in Fig. 8. The strain values of stress distribution were arbitrarily chosen as the value corresponding to elastic deformation. These values are for the whole system, in comparison to values only for interface region shown earlier in Fig. 8. 


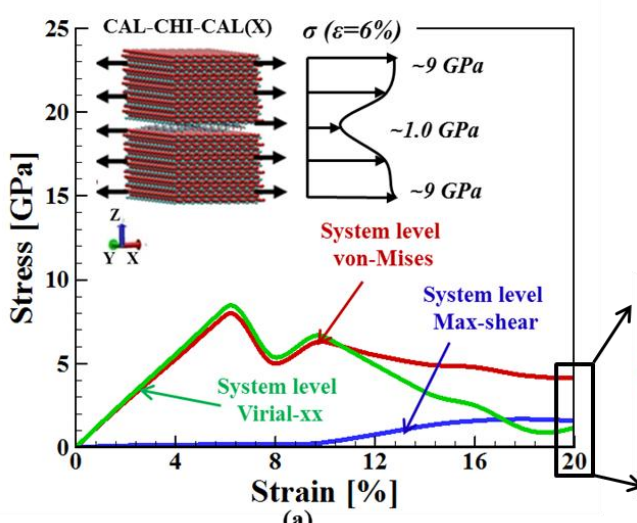

(a)

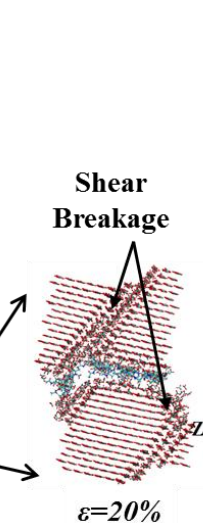

$\varepsilon=20 \%$
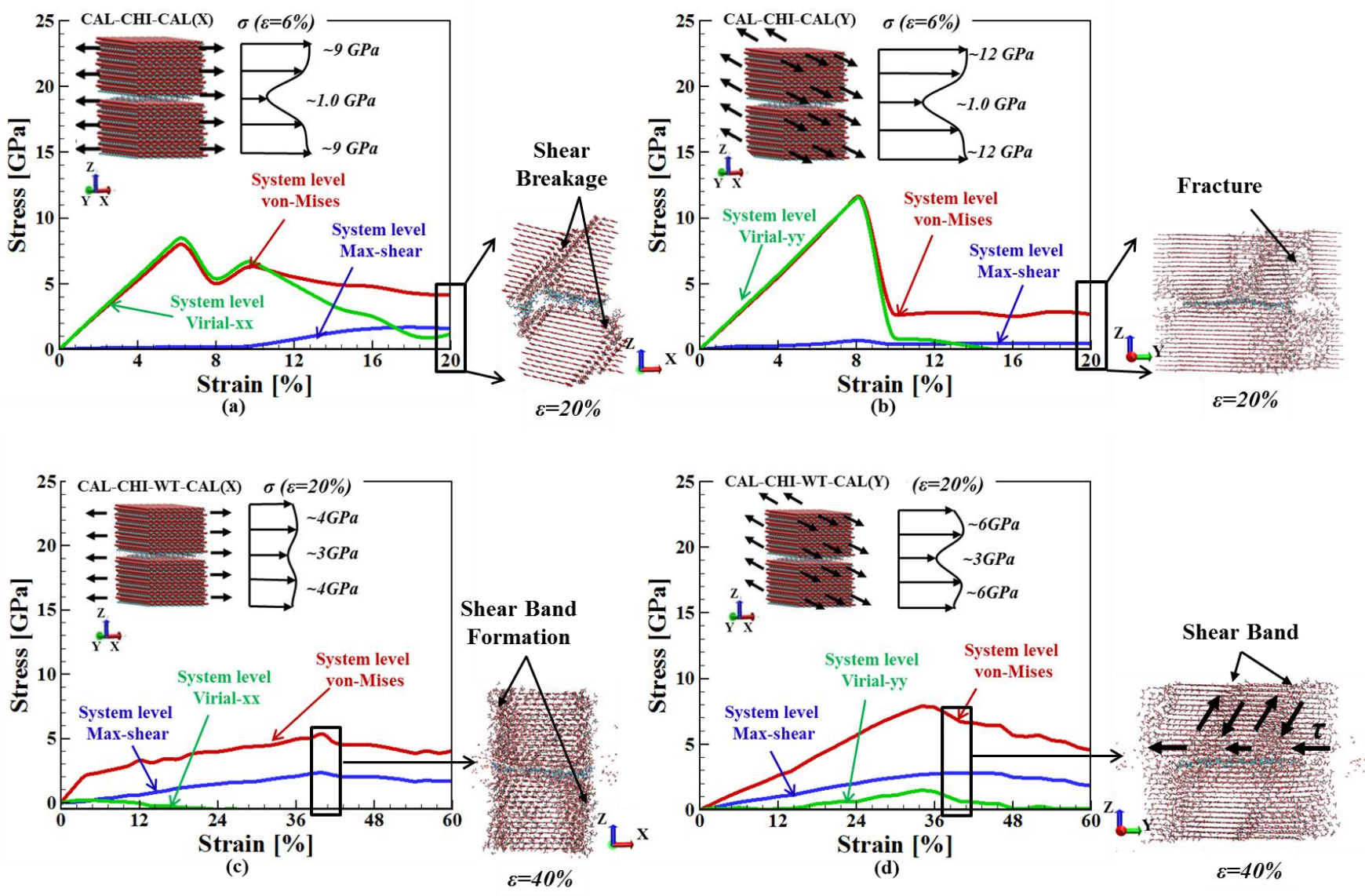

Figure 9. Plot of the overall (system level) von-Mises (red), virial (green), and maximum shear stress (blue) as a function of strain combined with the stress distribution based on the pressure profile calculation along the $z$-axis for each system (a) CAL-CHI-CAL loaded in $x$-axis, i.e. fibril direction, (b) CAL-CHI-CAL loaded in $y$-direction, i.e. transverse fibril direction, (c) CAL-CHIWT-CAL loaded in $x$-axis, i.e. fibril direction, (d) CAL-CHI-WT-CAL loaded in $y$-direction, i.e. transverse fibril direction.

Based on the pressure distribution plots it is clear that hydrated material systems undergo higher stress inside the interface region $(\sim 3.0 \mathrm{GPa})$ while un-hydrated systems undergo lower interface stress $(\sim 1.0 \mathrm{GPa})$. Furthermore, the gradient of the stress between the interface region and top/bottom regions is different. The overall maximum principal shear stress and interface maximum principal shear stress values are close to each other. The contribution of shear stress to material deformation can be quantified by the ratio of the shear stress to the von-Mises stress, 
denoted as $\delta$. Qualitative analysis shows that $\delta_{b}<\delta_{a}<\delta_{d}<\delta_{c}$, Fig. 9. The addition of water is observed to significantly affect the stress distribution of the material systems which further leads to significant strain hardening effect on the interfacial systems, where shear stress is the dominant of the mechanical behavior of the material systems, Fig. 9-(c) and (d). Therefore, higher shear contribution can be correlated to higher ductility in material deformation behavior. To further understand the effect of interfaces on the overall mechanical behavior of the systems, we use VMD [44] to show the snapshots of deformation of the interfacial systems combined with corresponding stress-strain curve, shown left to each plot in Fig. 9. In the case of CAL-CHI-CAL loaded in x-axis, it is clear to observe the plane of shear breakage of the CAL platelet at the $20 \%$ strain level. The shear breakage leads to the drop of the axial stress but an increase in the shear stress which enhances the contribution of the shear stress, shown in Fig. 9-(a). However, the interfacial level shear is not the main contributor to the system level shear stress, shown in Fig. 8-(a), since the shear breakage occurs in CAL minerals not in CHI chains. In the case of CALCHI-CAL loaded in y-axis, the failure mode is observed as the fracture of the CAL mineral at the peak strain, where both of the system level and interface level shear stress is much lower comparing to the von-Mises, Fig. 9-(b). The anisotropy of the deformation modes between these two cases could be attributed to two factors. First, the CAL crystal structure determines the plane where dislocations (i.e. slip or twinning) are easy to initiate. Second, the significant mismatch of the axial mechanical properties between CHI ( 28 GPa [22]) and CAL ( 140 GPa [50-52]) along $\mathrm{x}$-axis can lead to the coupled uniaxial and shear deformation, while it is less significant along $\mathrm{y}$ axis (CHI $\sim 119 \mathrm{GPa}$ [22] vs CAL $\sim 140 \mathrm{GPa}$ [50-52]). This is verified by comparing Fig. 8-(b) with Fig. 9-(b), where both of interface and system level Virial (uniaxial) stress reaches the maximum at the same strain level. In the hydrated conditions, the stiffened CHI, outlined earlier, 
significantly affect the deformation mechanisms. When loaded along x-axis, the stiffened interface behaved as the stopper which impede the formation and propagation of the slip (or twinning) systems in CAL mineral [58]. Instead, the shear band is trying to format at some point. The relevance of the shear banding phenomena is that they precede failure and energy is stored through the shear band formation [59], which explains the strain hardening effect observed in the stress-strain curve, Fig. 9-(c). The shear band is shown clearly when loaded along y-axis, Fig. 9(d), and arrows in the snapshot indicate the shear flow. The stiffened hydrated CHI interface behaves like a stiffer inclusion under the shear loading [59], the strain is localized through the shear band and stress is transferred by the shearing interaction through the interface $[58,59]$. This significant shear enhancement effect agrees with both of interface and system level stressstrain curve shown in Fig. 8-(d) and Fig. 9-(d). The anisotropy of the hydrated interface is not as significant as that shown in the un-hydrated conditions since shearing dominates the overall deformation and shear modulus of CHI in $\mathrm{x}-\mathrm{z}$ and $\mathrm{y}-\mathrm{z}$ plane $(\sim 5 \mathrm{GPa}$ and $\sim 8 \mathrm{GPa}$ [22], respectively) are on the same level.

Based on the above discussion, it can therefore be concluded that the interfacial shear, especially in hydrated condition, behaves like a stopper, mitigating the catastrophic failure of the material.

\section{\$3.2 Interfacial Shear Strength}

As outlined above, the organic phases carry shear load and this shear interaction plays a significant role in determining the overall interfacial mechanical behavior. The SMD simulations were performed based on the framework outlined earlier in section 2 to further study the interaction between the inorganic and organic phases. Fig. 10-(a) displays the force as a function

of displacement of chitin based materials obtained from the SMD simulation with $k=100$ 
$\mathrm{kcal} / \mathrm{mol} / \mathrm{A}^{2}$, and $v=0.4 \mathrm{~A} / \mathrm{ps}$. The following conclusions were reached based on the characterization of the sliding process using the four stages described in the section 2.3.1.

(1) The yield shear stress of CAL-CAL (red curve in Fig. 10-(a)) is much higher than that of the systems with organic interfaces or water interfaces. The interfacial mechanics between the inorganic crystals is affected by ionic bonding (electrostatic potential energy) and Van der Waals (VDW) forces. The interfacial mechanics between inorganicorganic interfaces primarily comes from the hydrogen bond (H-bond). The ionic bonding is shown to be much stronger (could be hundreds of $\mathrm{kJ} / \mathrm{mol}$ ) than the $\mathrm{H}$-bond (5 to 30 $\mathrm{kJ} / \mathrm{mol}[60]$ ). This leads to higher yield stress of the inorganic-inorganic interfacial systems and the higher strength of the inorganic-inorganic materials discussed in section

\subsection{1.}

(2) During the transition stage of the sliding process (the third stage described in section 2.3.1), the force decreases to the steady state force (failure stress, $\tau_{\mathrm{F}}$ ) from the critical force (yield stress, $\tau_{\mathrm{Y}}$, the breakage of the non-bonded energy between the interfaces occurs) in the case of CAL-CAL (red curve in Fig.10-(a)), where $\tau_{\mathrm{Y}}>\tau_{\mathrm{F}}$, and the sliding process shows the behavior like brittle material. While in the cases of organic interfaces, or in the case of interfaces with water, where $\tau_{Y}<\tau_{\mathrm{F}}$, the force slowly increases from the critical force (yield stress, $\tau_{Y}$, indicated using the red dot in Fig. 10-(a)), initiating the sliding between the SMD crystal and the organic phases or water phase, to the steady state force (failure stress, $\tau_{\mathrm{F}}$, indicated using the red dotted line in Fig. 10-(a)). The plastic shear deformation between the organic phases and the inorganic phases occurs and the sliding process behaves like the ductile material. The serrations in the force (failure stress) curve during the steady state deformation has been earlier studied using multiple 
models of the atomic scale friction, [47]. The transition stage of the plastic shear deformation is further characterized using a visco-plastic model of [27] in the next section.

(3) Water affects the yield and the failure shear stress. On one hand, water acts as extra cross-liking bridge, [29], at the interfaces by leading more hydrogen atoms participating in H-bond formation and increases the shear by increasing the interfacial contact area. On the other hand, it has been reported, [29, 61] , that water may act as lubricating agent between the interfaces during the deformation. These two factors compete with each other leading to the effect of water on the interfacial sliding process, Fig. 10-(a), i.e. water slightly increases the failure stress, $\tau_{\mathrm{F}}$. More accurate calculation in next section shows that water may lead to increase or decrease of the yield stress, $\tau_{\mathrm{Y}}$.

(4) The loading rate (i.e. pulling velocity in SMD simulation, v) dependency of the interface strength (measured as critical force, $\tau$ ) obtained from the simulation is quite sensitive, i.e. the critical force decreases sharply as the loading rate decreases [62-65], observed in Figure 10-(b). Due to the computational capability of SMD simulation, different pulling velocities were applied to the CAL-CHI-CAL system, and the critical force within the range of experimental pulling velocities (i.e. $10^{-13} \sim 10^{-7} \mathrm{~m} / \mathrm{s}[66,67]$ ) was determined using the extrapolation model [62-65], Eq. (6), Fig. 10-(c),

$$
F=A v-A v e^{(-B / \sqrt{v})}+C
$$

Where A, B and C are coefficients interpreted in terms of activation energy and activation volume [62-65]. The extrapolation model, Eq. (6), captures the significant loading rate dependency of the SMD simulations and bridges the SMD interface strength results with the continuum studies of the materials based on the experimental loading rate. 


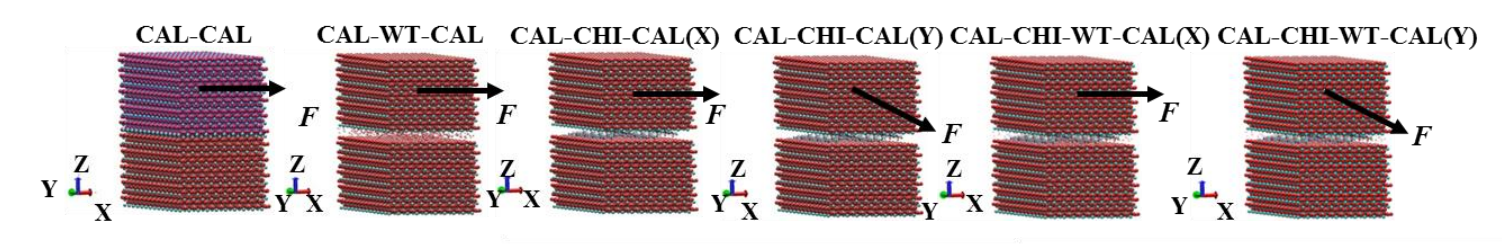

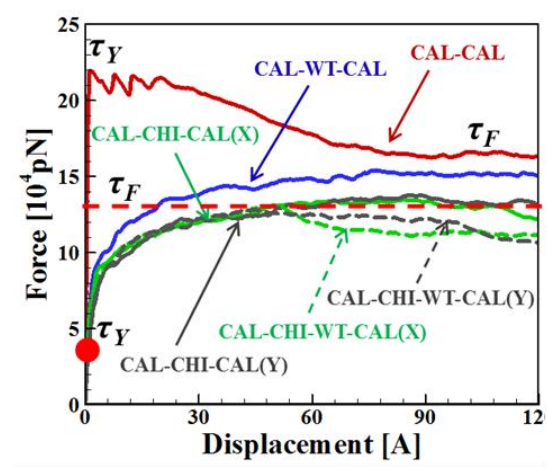

(a)

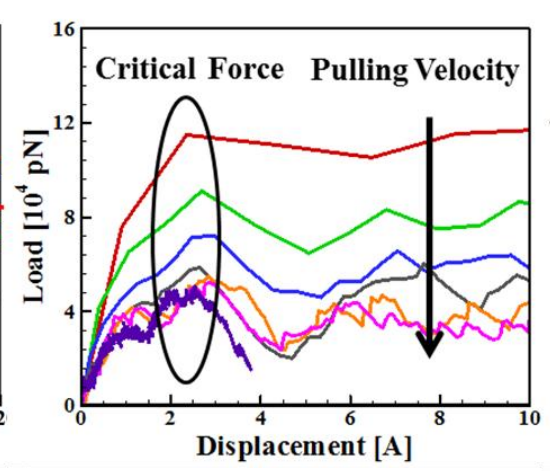

(b)

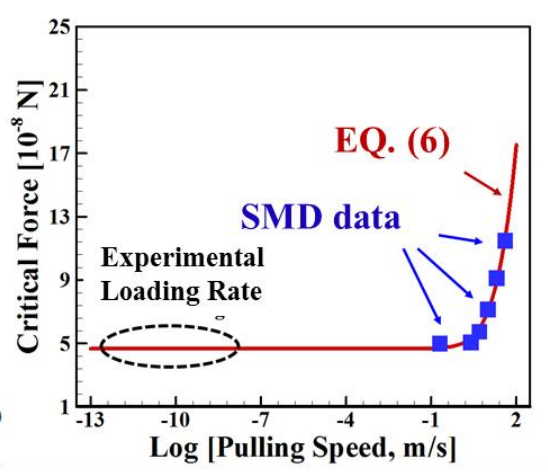

(c)

Figure 10. (a) SMD force as a function of displacement for different systems, (b) SMD force as a function of displacement for the system CAL-CHI-CAL loaded along x-axis at different loading rate, (c) extrapolation model to downscale the critical force measured by SMD simulations as a function of loading rate

The shear viscosity of the interfacial systems is calculated using a visco-plastic interfacial sliding model to further characterize the plastic shear deformation of the interfacial systems, and to study the effect of the interfacial strength on the deformation mechanism of the materials.

\section{§3.3 Interfacial Shear Deformation}

The yield shear stress, $\tau_{Y}$, is the yield stress of the sliding process described earlier and calculated using the visco-plastic interfacial sliding model introduced in section 2.3. The failure stress, $\tau_{F}$, is the frictional force during the steady state interfacial sliding process, and is obtained from the results of the SMD simulations present in last section. 


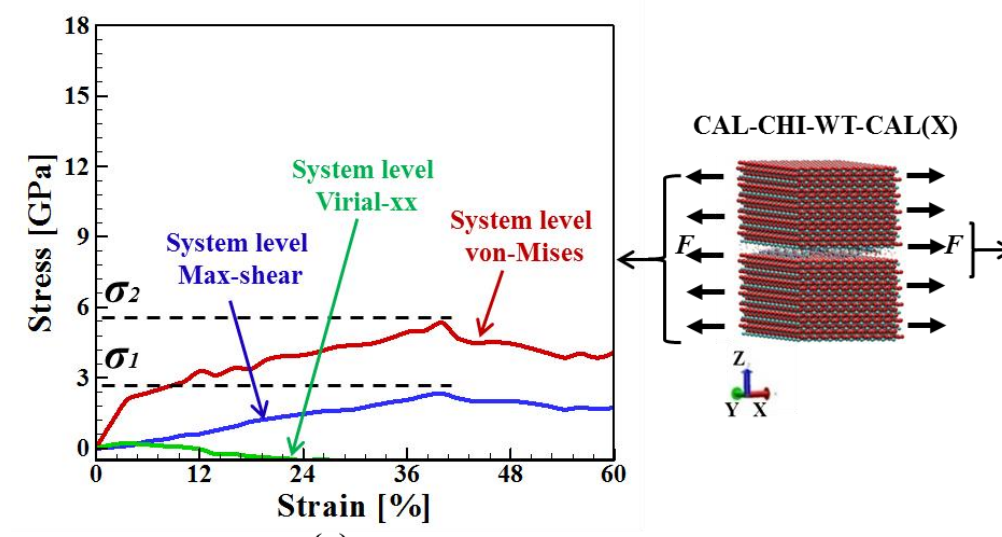

(a)

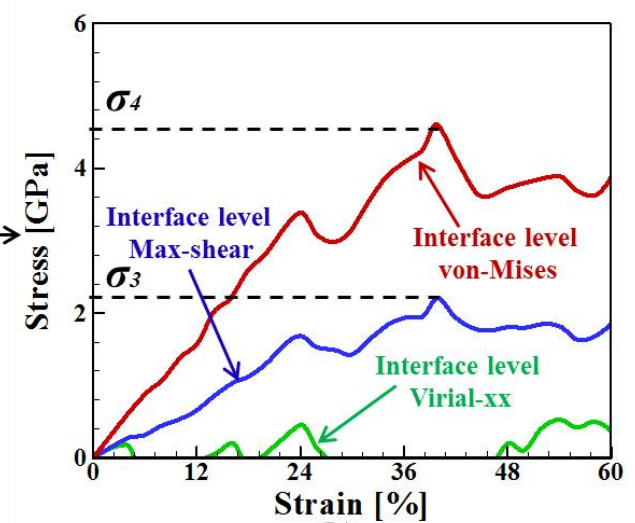

(b)

Figure 11. (a) plot of the system level von-Mises, virial and maximum shear stress-strain curve for the system CAL-CHI-WT-CAL loaded in $x$-axis, where, $\sigma_{1}$ is the peak stress of the maxshear) is the interfacial shear strength of the material system, $\sigma_{2}$ (peak stress of the von-Mises) is the mechanical strength of the material system, (b) plot of the interface level von-Mises, virial and maximum shear stress-strain curve for the system CAL-CHI-WT-CAL loaded in $x$-axis, where, $\sigma_{3}$ (peak stress of the max-shear) is the shear strength of the organic phase of the corresponding material system, $\sigma_{4}$ (peak stress of the von-Mises) is the mechanical strength of the organic phase of the corresponding material system.

As an example, Fig. 11 displays the von-Mises, virial and the maximum shear stress as a function of strain for the CAL-CHI-WT-CHI system loaded along $x$-axis. Fig. 11(a) shows stress values for the overall system (system level) and Fig. 11-(b), shows stress values for the interface region (interface level). The interfacial shear strength of the material system, $\sigma_{l}$, is defined as the peak stress of the system level maximum shear curve (blue colored) in Fig. 11-(a). The mechanical strength of the material system, $\sigma_{2}$, is defined as the peak stress of the system level von-Mises curve (red colored) in Fig. 11(a). The shear strength of organic phase (i.e. CHI) in the material system, $\sigma_{3}$, is defined as the peak stress of the interface level maximum shear curve (blue colored) in Fig. 11-(b). The mechanical strength of organic phase (i.e. CHI) in the material system, $\sigma_{4}$, is defined as the peak stress of the interface level von-Mises curve (red colored) in Fig. 11(b). These definitions will be used in the following analyses. 


\section{\$3.4 Viscous Interfaces}

Fig. 12 summarizes all of the mechanical strengths for the analyzed systems. The lower and upper bound of the shear strength of material systems with organic interfaces with or without presence of water could be defined by the yield shear stress, $\tau_{Y}$, and the failure stress, $\tau_{F}$. These are used to characterize plastic shear deformation, where $\tau_{\mathrm{Y}}<\tau_{\mathrm{F}}$. Figure 12 displaying yield shear stress $\left(\tau_{Y}\right)$, failure stress $\left(\tau_{F}\right)$, interfacial shear strength of the material system $\left(\sigma_{l}\right)$, shear strength of the organic phases (or WT) $\left(\sigma_{3}\right)$, mechanical strength of the organic phases (or WT) $\left(\sigma_{4}\right)$, as a function of the interfacial components of chitin based materials confirms it. The shear strength of organic phase (green line, $\sigma_{3}$ ) lies around the lower bound line of the yield shear stress (blue dotted line, $\left.\tau_{Y}\right)$. As discussed earlier in section 3.1, the organic phase is the main contributor of the interfacial shear strength, which is again confirmed in Fig. 12 where the interfacial shear strength (grey line, $\sigma_{1}$ ) matches closely with the shear strength of organic phases (green line, $\sigma_{3}$ ). However, it is always below the upper bound line defined by the failure stress (red dotted line, $\left.\tau_{F}\right)$ which is defined as the stress that initiates "catastrophic failure" of the interfaces. The mechanical strength of the organic phases (yellow line, $\sigma_{4}$ ) usually lies between the lower and upper bound line because shear deformation is usually the main contributor to the mechanical behavior of organic phases. The points above the upper bound are in the cases of hydrated organic interfaces (i.e. TC-WT and CHI-WT). This can be attributed to much higher contribution of the shear interaction to the overall behavior as well as higher shear viscosity of the interfacial material systems. 


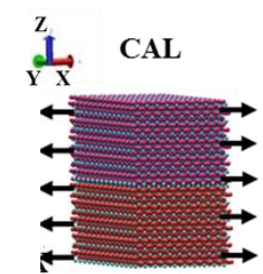

d 9.4 A

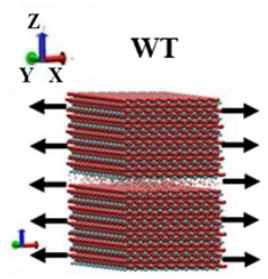

d $\sim 9.8 A$

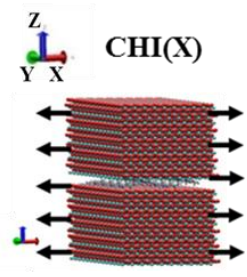

$d \sim 9.5 A$

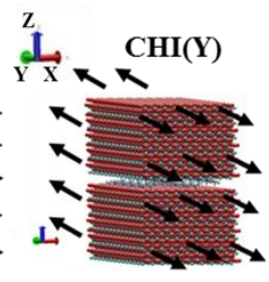

$d \sim 9.5 A$

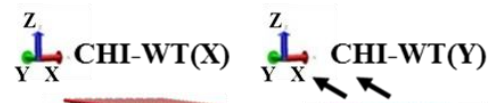

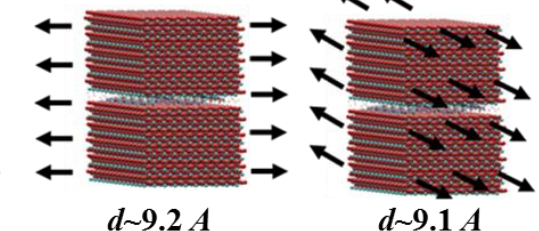

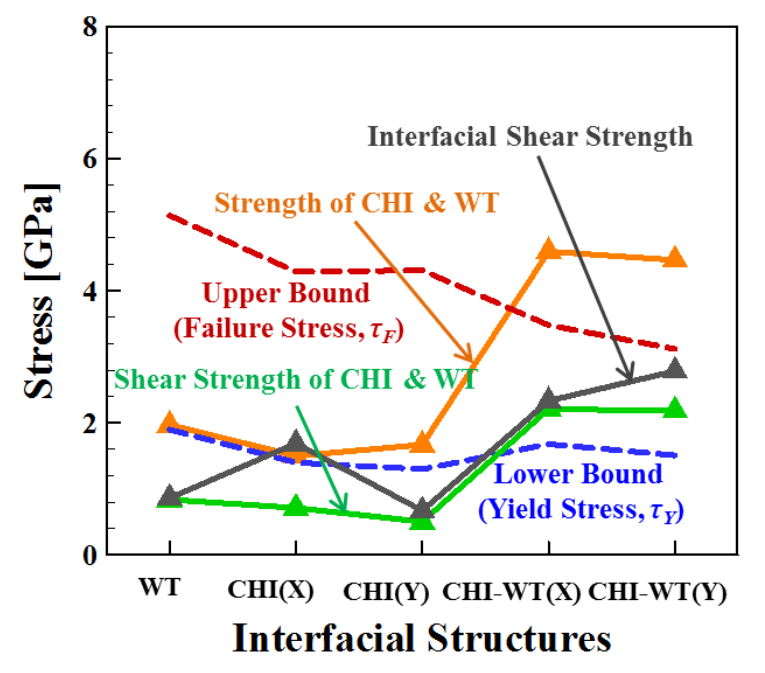

Figure 12. Stress as function of interfacial structures for chitin based materials

\section{\$3.5 Analytical Characterization of Viscous Interfaces}

The material model presented in this paper consists of $\alpha$-chitin layers in between two layers of calcite. The calcite layers are same throughout different systems. The composition of the interface layers vary in different structures. In the present paper, a comparison is drawn between interfaces with and without presence of water molecules in $\mathrm{x}$ and $\mathrm{y}$ direction. Viscosity calculations in previous sections were performed based on MD Interfacial sliding model. It takes into account shear force and displacement of mass center of the deformed model. The velocities were calculated by taking the numerical derivative of displacement. In this section, an alternate approach is used to calculate viscosity parameters from the stress-strain data of the systems under investigation to further validate the results. A simple model combining Kelvin Voigt and 
Maxwell model is used to represent the viscous interfaces and calcite layers. These models has been used by several researchers to model viscous behavior of bones and tissues [68-71]. It has been reported $[72,73]$ that the viscous adhesive behavior of bio interfaces is dominated by sacrificial bonds between $\mathrm{Ca}^{2+}$ ions and organic fibers. This "glue" interface promotes energy dissipation during deformation of the material and this mechanism works better when more positive ions, such as $\mathrm{Ca}^{2+}$, are involved [72]. These viscous hydrated interfaces can act as the source of the macroscopic phenomenon of biomaterial viscoelasticity, [30, 31]. Presence of the interface with higher viscosity is the main contributor of the toughening mechanisms to prevent catastrophic failure. At the nanoscale, the interfaces reduce the stiffness but improve the toughness of the biomaterial by affecting the stress distribution (more uniform along $z$ coordination), enhancing the shear contribution to the overall mechanical behavior and promoting energy dissipation required for viscoelastic deformation of the organic phases [55].

Figure 13 shows the representation of the Kelvin Voigt and Maxwell model. It is assumed that calcite layers only contribute to the elastic part. The interface region contributes to both elastic and viscous part of the system. Stress and strain data for the analyzed models was calculated from the MD simulations. Modulus and viscosity for the MD simulation results given in Table 1 are calculated from the stress-strain curves given in Figure 9 and equation 4. 

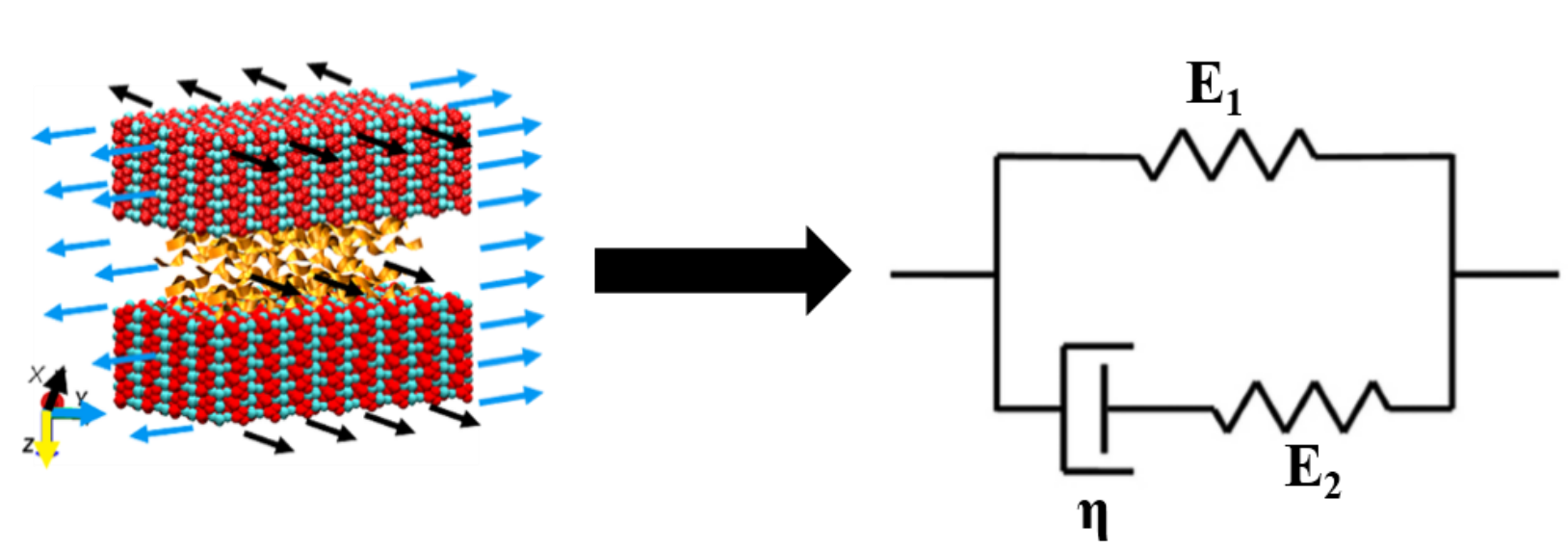

Figure 13: Schematic of Calcite and Chitin interface based on the Kelvin Voigt and Maxwell model

Spring element with stiffness $E_{1}$ in Fig. 13 represents calcite blocks. Spring element $E_{2}$ is stiffness of viscous interface and $\eta$ represents viscosity of the interface. $\eta$ is defined as the loss factor used to quantify damping performance. Higher $\eta$ leads to more significant effect of the damping system on the deformation mechanism, more energy dissipation during the elastic deformation, more ductile behavior and lower effective stiffness of the material. Solving this model, stress-strain relation can be expressed as

$$
\sigma=-\left(\frac{\eta}{E_{2}}\right) \frac{d \sigma}{d t}+E_{1} * \varepsilon+\left(1+\frac{E_{1}}{E_{2}}\right) \eta \frac{d \varepsilon}{d t}
$$

Here, $\sigma$ is the stress, $\varepsilon$ is strain, $E_{1}$ is stiffness of calcite, $E_{2}$ is stiffness of chitin, $\eta$ is viscosity of $\alpha$-chitin interface. The stress-strain data from the analyzed models was fit into equation 7 to obtain values of parameters $E_{1}, E_{2}$ and $\eta$ of Kelvin Voigt and Maxwell system as given in Table 1. The values from both analytical model and from the MD simulations shows the same trend in viscosity. The difference of one order of magnitude in MD viscosity values is because of the model used for the calculation. MD model only considers shear stress contribution while equation 7 considers overall stress-strain behavior of the material. 
Table 1 : Parameters calculated by Kelvin Voigt and Maxwell model and viscosity from MD simulations

\begin{tabular}{|c|c|c|c|c|c|}
\hline & \multicolumn{3}{|c|}{ Fit Parameters from Equation } & \multicolumn{2}{c|}{ MD Simulations Results } \\
\cline { 2 - 6 } & $\begin{array}{c}\text { Calcite - } \\
\text { Modulus (GPa) }\end{array}$ & $\begin{array}{c}\text { a-chitin- } \\
\text { Modulus (GPa) }\end{array}$ & $\begin{array}{c}\text { Viscosity } \\
(\mathrm{Pa} \mathrm{s})\end{array}$ & $\begin{array}{c}\text { Modulus } \\
(\mathrm{GPa})\end{array}$ & $\begin{array}{c}\text { Viscosity } \\
(\mathrm{Pa} \text { s) }\end{array}$ \\
\hline cal-chi- & 130 & 150 & 0.0036 & 130.90 & 0.0570 \\
\hline cal-chi- & 140 & 15 & 0.0011 & 144.70 & 0.0580 \\
\hline cal-chi-wt- & 12 & 0.15 & 0.0025 & 10.02 & 0.0460 \\
\hline cal-chi-wt- & 23 & 1.50 & 0.0004 & 23.86 & 0.0480 \\
\hline
\end{tabular}

The viscosity values are plotted in Figs. 14 (a) and (b). It shows that viscosity of the system with water is lower as compared to the same system without presence of water in the interface. The model used to fit stress strain data is for the overall structure and it correlates well with the physical behavior. In the presence of water, the overall viscosity of the system would be less as expected. Figure 14 (a) shows that viscosity in the presence of water is less in x direction and Fig (b) shows the viscosity behavior of y directions. The deformation behavior is shear dominated in the presence of the water as explained in the section 3.1. This leads to a drop in the elastic modulus. Elastic modulus of calcite is in the range of actual elastic modulus values for calcium that further verifies our model. Since the fibers are oriented in one direction, they show higher modulus in one directions as compared to others. The viscosity values calculated in current study are similar to values calculated by Y. Ichikawa et al.[74]. We have calculated viscosity from two different approaches. The comparison of the results give us more confidence in the chosen model. 

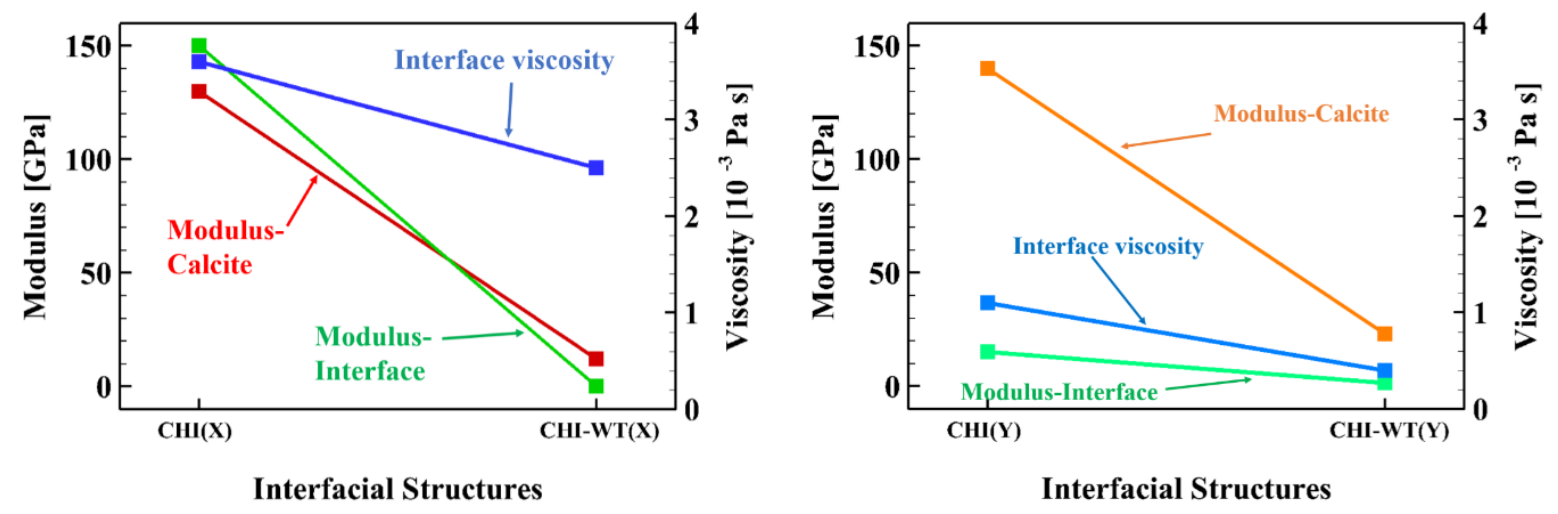

Figure 14 Modulus and viscosity of interfacial structures of chitin based materials in (a) X direction and (b) Y direction.

The analyses of organic-inorganic biomaterials (e.g. bone, marine exoskeleton, nacre etc.) based on the tension-shear-chain models [34-36] with organic fibers (e.g. chitin) are aligning in a direction parallel to the surface of inorganic crystals (e.g. calcite) (Fig. 2) pointed that the shear deformation between the soft matrix and inorganic crystals is the main stress transfer mechanism of such loaded biomaterials, and recent studies proposed that the layered water in crystal interfaces could be the source for biomaterial viscoelasticity [30, 31]. Therefore, the magnitude of viscosity reported in this study is a potentially important value to be used with analytical models to determine the biomaterial viscoelastic properties. Presence of water in interface decreases viscosity of the overall material as compared to interfaces without water. It also further effects the other elastic properties of material such as modulus and hardness.

\section{\$4 Conclusions}

Chitin-based interfacial supercells were analyzed for their mechanical properties using two different variants of classical MD simulation method. The plastic shear deformation behavior 
was characterized with a well known visco-plastic interfacial sliding model. The conclusions are summarized below.

(1) Inorganic phases in the material systems carry the uniaxial tensile loading while the organic phases carry primarily the shear loading.

(2) Organic interfacial systems exhibit plastic shear deformation, and the yield and failure shear stress define the lower and higher bound of the interfacial strength.

(3) Interfacial strength measured by SMD simulations shows a highly loading rate dependent behavior, and the overall behavior of this behavior can be captured using the extrapolation estimation with the SMD data.

(4) The addition of water enhances the contribution of interfacial shear to the overall mechanical behavior of the interfacial material systems, as well as decreases the shear viscosity between the organic and inorganic phases in biomaterials.

(5) At nanoscale, the (hydrated) organic-inorganic interfaces improve the toughness of the biomaterial systems by enhancing the contribution of the interfacial shear, introducing the viscous interfaces, and promoting energy dissipation required for viscoelastic deformation to prevent the catastrophic failure.

The observations and shear viscosity reported here provide important data and insights for formulating continuum relations for bio-composite materials with viscous interfaces. At this point it is important to remember that interfaces considered in this work are idealized in order to systematically delineate the influence of various structural factors.

\section{$\$ 5$ Disclosures}

There is no conflict of interest with any party reported in this work or otherwise. 


\section{§6 Acknowledgement}

This work was supported by National Science Foundation Grant CMMI1131112. 


\section{REFERENCES}

[1] Castner DG, Ratner BD. Biomedical surface science: Foundations to frontiers. Surface Science 2002;500:28-60.

[2] Al - Sawalmih A, Li C, Siegel S, Fabritius H, Yi S, Raabe D, et al. Microtexture and chitin/calcite orientation relationship in the mineralized exoskeleton of the American lobster. Advanced functional materials 2008;18:3307-14.

[3] Giraud-Guille M-M. Fine structure of the chitin-protein system in the crab cuticle. Tissue and Cell 1984;16:75-92.

[4] Giraud-Guille M-M, Chanzy H, Vuong R. Chitin crystals in arthropod cuticles revealed by diffraction contrast transmission electron microscopy. Journal of Structural Biology 1990;103:232-40.

[5] Roer R, Dillaman R. The structure and calcification of the crustacean cuticle. American Zoologist 1984;24:893-909.

[6] Raabe D, Al-Sawalmih A, Romano P, Sachs C, Brokmeier H, Yi S, et al. Structure and crystallographic texture of arthropod bio-composites. Materials Science Forum: Transtec Publications; 1999; 2005. p. 1665.

[7] Raabe D, Romano P, Sachs C, Fabritius H, Al-Sawalmih A, Yi S-B, et al. Microstructure and crystallographic texture of the chitin-protein network in the biological composite material of the exoskeleton of the lobster $<\mathrm{i}>$ Homarus americanus $</ \mathrm{i}>$. Materials Science and Engineering: A 2006;421:143-53.

[8] Raabe D, Sachs C, Romano P. The crustacean exoskeleton as an example of a structurally and mechanically graded biological nanocomposite material. Acta Materialia 2005;53:4281-92.

[9] Joffe I, Hepburn H, Nelson K, Green N. Mechanical properties of a crustacean exoskeleton. Comparative Biochemistry and Physiology Part A: Physiology 1975;50:545-9.

[10] Vincent JF, Wegst UG. Design and mechanical properties of insect cuticle. Arthropod Structure \& Development 2004;33:187-99.

[11] Sachs C, Fabritius H, Raabe D. Hardness and elastic properties of dehydrated cuticle from the lobster Homarus americanus obtained by nanoindentation. Journal of Materials Research 2006;21:1987-95.

[12] Sachs C, Fabritius H, Raabe D. Influence of microstructure on deformation anisotropy of mineralized cuticle from the lobster $<\mathrm{i}>$ Homarus americanus $</ \mathrm{i}>$. Journal of Structural Biology 2008;161:120-32.

[13] Fabritius HO, Sachs C, Triguero PR, Raabe D. Influence of Structural Principles on the Mechanics of a Biological Fiber - Based Composite Material with Hierarchical Organization: The Exoskeleton of the Lobster Homarus americanus. Advanced Materials 2009;21:391-400. 
[14] Gao HJ, Ji BH, Jager IL, Arzt E, Fratzl P. Materials become insensitive to flaws at nanoscale: Lessons from nature. Proceedings of the National Academy of Sciences of the United States of America 2003;100:5597-600.

[15] Gao HJ, Wang X. Mechanics of hierarchical adhesion structures of geckos. Mechanics of Materials 2005;37:275-85.

[16] Dubey DK, Tomar V. Understanding the influence of structural hierarchy and its coupling with chemical environment on the strength of idealized tropocollagen-hydroxyapatite biomaterials. Journal of the Mechanics and Physics of Solids 2009;57:1702-17

[17] Dubey DK, Tomar V. Role of the nanoscale interfacial arrangement in mechanical strength of tropocollagen-hydroxyapatite based hard biomaterials. Acta Biomaterialia 2009;5:2704-16.

[18] Dubey DK, Tomar V. The effect of tensile and compressive loading on the hierarchical strength of idealized tropocollagen-hydroxyapatite biomaterials as a function of the chemical environment. Journal of Physics-Condensed Matter 2009;21.

[19] Dubey DK, Tomar V. Role of hydroxyapatite crystal shape in nanoscale mechanical behavior of model tropocollagen-hydroxyapatite hard biomaterials. Materials Science \& Engineering C-Materials for Biological Applications 2009;29:2133-40.

[20] Jin K, Feng X, Xu Z. Mechanical Properties of Chitin-Protein Interfaces: A Molecular Dynamics Study. BioNanoScience 2013:1-9.

[21] Nikolov S, Fabritius H, Petrov M, Friák M, Lymperakis L, Sachs C, et al. Robustness and optimal use of design principles of arthropod exoskeletons studied by ab initio-based multiscale simulations. Journal of the Mechanical Behavior of Biomedical Materials 2011;4:129-45.

[22] Nikolov S, Petrov M, Lymperakis L, Friák M, Sachs C, Fabritius HO, et al. Revealing the Design Principles of High - Performance Biological Composites Using Ab initio and Multiscale Simulations: The Example of Lobster Cuticle. Advanced Materials 2010;22:519-26.

[23] Bhowmik R, Katti KS, Katti DR. Influence of mineral on the load deformation behavior of polymer in hydroxyapatite-polyacrylic acid nanocomposite biomaterials: A steered molecular dynamics study. Journal of nanoscience and nanotechnology 2008;8:2075-84.

[24] Bhowmik R, Katti KS, Katti DR. Mechanisms of load-deformation behavior of molecular collagen in hydroxyapatite-tropocollagen molecular system: steered molecular dynamics study. Journal of engineering mechanics 2009;135:413-21.

[25] Katti DR, Pradhan SM, Katti KS. Directional dependence of hydroxyapatite-collagen interactions on mechanics of collagen. Journal of Biomechanics 2010;43:1723-30.

[26] Lindahl E, Edholm O. Spatial and energetic-entropic decomposition of surface tension in lipid bilayers from molecular dynamics simulations. The Journal of chemical physics 2000;113:3882-93. 
[27] Frankland S, Harik V. Analysis of carbon nanotube pull-out from a polymer matrix. Surface Science 2003;525:L103-L8.

[28] Buehler MJ. Nature designs tough collagen: explaining the nanostructure of collagen fibrils. Proceedings of the National Academy of Sciences of the United States of America 2006;103:12285-90.

[29] Dubey DK, Tomar V. Ab Initio Investigation of Strain Dependent Atomistic Interactions at Two Tropocollagen-Hydroxyapatite Interfaces. Journal of Engineering Materials and Technology 2013;135:021015-.

[30] Shahidi M, Pichler B, Hellmich C. Viscous interfaces as source for material creep: a continuum micromechanics approach. European Journal of Mechanics-A/Solids 2014;45:41-58.

[31] Eberhardsteiner L, Hellmich C, Scheiner S. Layered water in crystal interfaces as source for bone viscoelasticity: arguments from a multiscale approach. Computer methods in biomechanics and biomedical engineering 2014;17:48-63.

[32] Qu T, Verma D, Shahidi M, Pichler B, Hellmich C, Tomar V. Mechanics of organicinorganic biointerfaces-Implications for strength and creep properties. MRS bulletin 2015;40:349-58.

[33] Qu T, Tomar V. Nanomechanics based investigation into interface-thermomechanics of collagen and chitin based biomaterials. 2014.

[34] An B, Zhao X, Zhang D. On the mechanical behavior of bio-inspired materials with nonself-similar hierarchy. Journal of the Mechanical Behavior of Biomedical Materials 2014;34:817.

[35] Zhang Z, Zhang Y-W, Gao H. On optimal hierarchy of load-bearing biological materials. Proceedings of the Royal Society B: Biological Sciences 2010:rspb20101093.

[36] Shuchun Z, Yueguang W. Effective elastic modulus of bone-like hierarchical materials. Acta Mechanica Solida Sinica 2007;20:198-205.

[37] Beckham GT, Crowley MF. Examination of the $\alpha$-chitin structure and decrystallization thermodynamics at the nanoscale. The Journal of Physical Chemistry B 2011;115:4516-22.

[38] Verma D, Tomar V. An Investigation into Mechanical Strength of Exoskeleton of Hydrothermal Vent Shrimp (Rimicaris Exoculata) and Shallow Water Shrimp (Pandalus Platyceros) at Elevated Temperatures. Materials Science and Engineering: C 2015.

[39] Minke R, Blackwell J. The structure of $\alpha$-chitin. Journal of molecular biology 1978;120:167-81.

[40] Sikorski P, Hori R, Wada M. Revisit of $\alpha$-chitin crystal structure using high resolution Xray diffraction data. Biomacromolecules 2009;10:1100-5. 
[41] Petrov M, Lymperakis L, Friák M, Neugebauer J. Ab Initio Based conformational study of the crystalline a - chitin. Biopolymers 2013;99:22-34.

[42] Maslen E, Streltsov V, Streltsova N. X-ray study of the electron density in calcite, CaCO3. Acta Crystallographica Section B: Structural Science 1993;49:636-41.

[43] Phillips JC, Braun R, Wang W, Gumbart J, Tajkhorshid E, Villa E, et al. Scalable molecular dynamics with NAMD. Journal of Computational Chemistry 2005;26:1781-802.

[44] Humphrey W, Dalke A, Schulten K. VMD: visual molecular dynamics. Journal of molecular graphics 1996;14:33-8.

[45] Ponder JW, Case DA. Force fields for protein simulations. Protein Simulations. San Diego: Academic Press Inc; 2003. p. 27-85.

[46] Vanommeslaeghe K, Hatcher E, Acharya C, Kundu S, Zhong S, Shim J, et al. CHARMM general force field: A force field for drug-like molecules compatible with the CHARMM allatom additive biological force fields. Journal of Computational Chemistry 2010;31:671-90.

[47] Singer I. Friction and energy dissipation at the atomic scale: A review. Journal of Vacuum Science \& Technology A 1994;12:2605-16.

[48] Shen M, Guan J, Xu L, Yu Y, He J, Jones GW, et al. Steered molecular dynamics simulations on the binding of the appendant structure and helix- $\beta 2$ in domain-swapped human cystatin C dimer. Journal of Biomolecular Structure and Dynamics 2012;30:652-61.

[49] Lelievre F, Bernache-Assollant D, Chartier T. Influence of powder characteristics on the rheological behaviour of hydroxyapatite slurries. Journal of Materials Science: Materials in Medicine 1996;7:489-94.

[50] Bhimasenachar J. Elastic constants of calcite and sodium nitrate. Proceedings Mathematical Sciences 1945;22:199-208.

[51] Peselnick L, Robie RA. Elastic constants of calcite. Journal of Applied Physics 1962;33:2889-92.

[52] Dandekar DP. Variation in the elastic constants of calcite with temperature. Journal of Applied Physics 1968;39:3694-9.

[53] Gupta HS, Seto J, Wagermaier W, Zaslansky P, Boesecke P, Fratzl P. Cooperative deformation of mineral and collagen in bone at the nanoscale. Proceedings of the National Academy of Sciences of the United States of America 2006;103:17741-6.

[54] Seto J, Gupta HS, Zaslansky P, Wagner HD, Fratzl P. Tough lessons from bone: extreme mechanical anisotropy at the mesoscale. Advanced functional materials 2008;18:1905-11.

[55] Meyers MA, McKittrick J, Chen P-Y. Structural biological materials: critical mechanicsmaterials connections. Science 2013;339:773-9. 
[56] Gupta H, Seto J, Krauss S, Boesecke P, Screen H. In situ multi-level analysis of viscoelastic deformation mechanisms in tendon collagen. Journal of Structural Biology 2010;169:183-91.

[57] Yamamoto Y, Nishimura T, Saito T, Kato T. CaCO3/chitin-whisker hybrids: formation of $\mathrm{CaCO} 3$ crystals in chitin-based liquid-crystalline suspension. Polymer journal 2010;42:583-6.

[58] Parisot SF-R. Material crystal plasticity and deformation twinning. Universitae Politecnico di Torino 2000:99.

[59] Bigoni D. Nonlinear solid mechanics: bifurcation theory and material instability: Cambridge University Press; 2012.

[60] Steiner T. The hydrogen bond in the solid state. Angewandte Chemie International Edition 2002;41:48-76.

[61] Zhang D, Chippada U, Jordan K. Effect of the structural water on the mechanical properties of collagen-like microfibrils: a molecular dynamics study. Annals of Biomedical Engineering 2007;35:1216-30.

[62] Buehler MJ. Fracture mechanics of protein materials Materials Today 2007;10:46-58.

[63] Rico F, Gonzalez L, Casuso I, Puig-Vidal M, Scheuring S. High-speed force spectroscopy unfolds titin at the velocity of molecular dynamics simulations. Science 2013;342:741-3.

[64] Hummer G, Szabo A. Kinetics from nonequilibrium single-molecule pulling experiments. Biophysical journal 2003;85:5-15.

[65] Gergely C, Voegel J-C, Schaaf P, Senger B, Maaloum M, Hörber J, et al. Unbinding process of adsorbed proteins under external stress studied by atomic force microscopy spectroscopy. Proceedings of the National Academy of Sciences 2000;97:10802-7.

[66] Dealy JM, Wang J. Melt rheology and its applications in the plastics industry: Springer; 2013.

[67] Franck A. Understanding Rheology of Thermoplastic Polymers. TA Instruments 2004.

[68] Gautieri A, Vesentini S, Redaelli A, Ballarini R. Modeling and measuring visco-elastic properties: From collagen molecules to collagen fibrils. International Journal of Non-Linear Mechanics 2013;56:25-33.

[69] Gautieri A, Vesentini S, Redaelli A, Buehler MJ. Viscoelastic properties of model segments of collagen molecules. Matrix Biology 2012;31:141-9.

[70] Korhonen RK, Saarakkala S. Biomechanics and modeling of skeletal soft tissues. 2011.

[71] Clausen AH, Polanco-Loria M, Berstad T, Hopperstad OS. A constitutive model for thermoplastics with some applications. 8th European LS-DYNA users conference2011. 
[72] Hansma PK, Fantner GE, Kindt JH, Thurner PJ, Schitter G, Turner PJ, et al. Sacrificial bonds in the interfibrillar matrix of bone. Journal of Musculoskeletal and Neuronal Interactions 2005;5:313.

[73] Thormann E, Mizuno H, Jansson K, Hedin N, Fernández MS, Arias JL, et al. Embedded proteins and sacrificial bonds provide the strong adhesive properties of gastroliths. Nanoscale 2012;4:3910-6.

[74] Ichikawa Y, Kawamura K, Fujii N, Nattavut T. Molecular dynamics and multiscale homogenization analysis of seepage/diffusion problem in bentonite clay. International journal for numerical methods in engineering 2002;54:1717-49. 


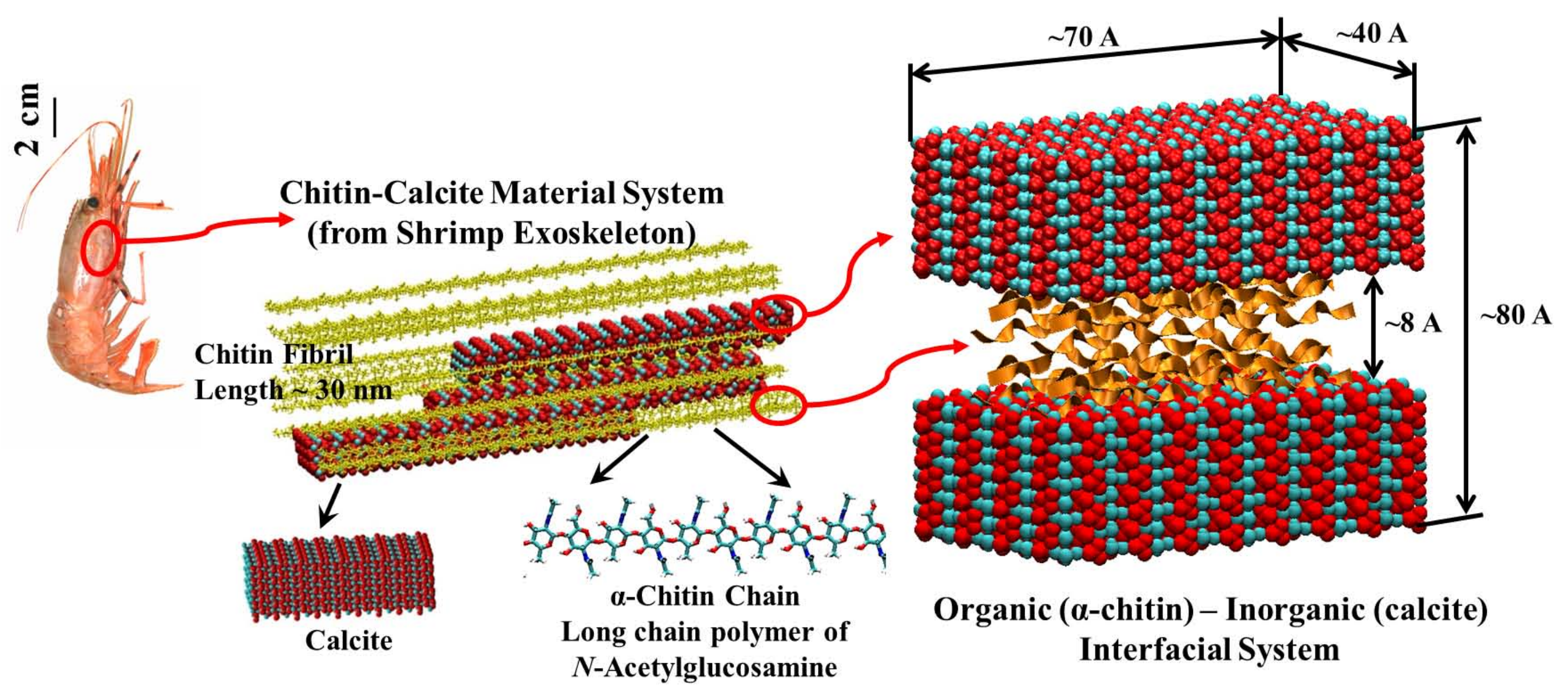

